\title{
Storage ring two-color free-electron laser
}

\author{
J. Yan, ${ }^{1,{ }^{*}}$ H. Hao, ${ }^{1}$ J. Y. Li ${ }^{2}$ S. F. Mikhailov, ${ }^{1}$ V. G. Popov, ${ }^{1}$ N. A. Vinokurov, ${ }^{3}$ S. Huang, ${ }^{4}$ J. Wu, ${ }^{5}$ \\ S. Günster, ${ }^{6}$ and Y. K. Wu ${ }^{1, \dagger}$ \\ ${ }^{1}$ FEL Laboratory, TUNL and Department of Physics, Duke University, Durham, \\ North Carolina 27708-0319, USA \\ ${ }^{2}$ NSRL, University of Science and Techonology of China, Hefei, Anhui 230029, China \\ ${ }^{3}$ Budker Institute of Nuclear Physics, Novosibirsk 630090, Russia \\ ${ }^{4}$ Institute of Heavy Ion Physics, School of Physics, Peking University, Beijing 100871, China \\ ${ }^{5}$ SLAC National Accelerator Laboratory, Stanford University, Stanford, California 94309, USA \\ ${ }^{6}$ Laser Zentrum Hannover e.V., Hollerithallee 8, 30419 Hannover, Germany
}

(Received 10 March 2016; published 5 July 2016)

\begin{abstract}
We report a systematic experimental study of a storage ring two-color free-electron laser (FEL) operating simultaneously in the infrared (IR) and ultraviolet (UV) wavelength regions. The two-color FEL lasing has been realized using a pair of dual-band high-reflectivity FEL mirrors with two different undulator configurations. We have demonstrated independent wavelength tuning in a wide range for each lasing color, as well as harmonically locked wavelength tuning when the UV lasing occurs at the second harmonic of the IR lasing. Precise power control of two-color lasing with good power stability has also been achieved. In addition, the impact of the degradation of FEL mirrors on the two-color FEL operation is reported. Furthermore, we have investigated the temporal structures of the two-color FEL beams, showing simultaneous two-color micropulses with their intensity modulations displayed as FEL macropulses.
\end{abstract}

DOI: 10.1103/PhysRevAccelBeams.19.070701

\section{INTRODUCTION}

Lasers operated with multiple colors have been developed over the last half century with a number of applications in physics research. For example, two optical pulses with different wavelengths but controllable time delay can be used in pump-probe spectroscopy to measure the fast dynamics of the system under investigation [1-3]. Two spatially and temporally overlapped laser beams with two colors can be employed in coherent anti-Stokes Raman spectroscopy to study molecular vibrations through nonlinear interactions with samples $[4,5]$. Some other applications of multicolor lasers include excited-state spectroscopy $[6,7]$ and photomixing processes for terahertz radiation generation $[8,9]$. The multicolor lasers with good collinearity are particularly important in research since the laser beams of different colors can be copropagated over a long distance, collimated and focused simultaneously. The typical approach to realize simultaneous multicolor lasing is using a dispersive or diffractive wavelength filter such as a prism or grating, either intracavity or in an external feedback cavity. Such a technology has been implemented in conventional lasers with different gain media such as dye [10-13],

\footnotetext{
;junyan@fel.duke.edu

wu@fel.duke.edu
}

Published by the American Physical Society under the terms of the Creative Commons Attribution 3.0 License. Further distribution of this work must maintain attribution to the author(s) and the published article's title, journal citation, and DOI. solid-state [14-17], semiconductor [18,19], and fiber [20-22]. However, the wavelength tunability of these lasers is limited by the bandwidth of the gain medium.

Since the theoretical prediction [23] and the first experimental demonstration [24] by Madey in the 1970s, free-electron lasers (FELs) have seen great development over the past few decades, and have become increasingly attractive light sources in a number of research areas. A common low-gain oscillator FEL uses an optical cavity to trap and amplify electron beam radiation. The oscillator FEL can be driven either by an electron storage ring or a linac. Oscillator FELs mainly operate in the spectral region from IR to vacuum UV. Another FEL configuration, the high-gain single-pass FEL, is mainly driven by linacs and does not use an optical cavity. In these FELs, the amplification of the FEL beam is realized in a single pass via the interaction between the electron beam and its radiation in a long undulator array [25,26] or with an external laser [27,28]. Single-pass FELs are now used as highperformance coherent light sources in the extreme UV and $\mathrm{X}$-ray regimes. The natural advantages of an FEL such as its broadband gain medium (an electron beam) and the copropagation of electron and light beams make an FEL an excellent device for multicolor lasing with good wavelength tunability and collinearity. The first multicolor FEL lasing was observed experimentally on an oscillator FEL with the optical klystron configuration on the VEPP-3 storage ring $[29,30]$. Later on some other storage ring based FELs such as super ACO FEL [31] also reported successful multicolor FEL operations with the use of optical klystrons. 
With this FEL configuration, the wavelength separations between the multiple wavelengths are limited by the bandwidth of undulator spontaneous radiation. In the last two decades, multicolor FEL operations have been widely developed and realized with several linac based FELs. In a typical linac based single-pass two-color FEL, a shared electron beam is employed to drive FEL lasing at two different wavelengths using two sets of undulators with different magnetic field strengths [32-34]. In a linac based oscillator FEL, two-color lasing also requires the FEL mirrors to have high reflectivity at these lasing wavelengths. For example, an IR oscillator FEL [35-37] with a relatively high gain can take advantage of the availability of broadband mirrors in the mid-infrared region.

Two-color lasing is more challenging for a storage ring FEL with a relatively low gain in the spectral region from near IR to vacuum UV. This difficulty can be overcome by using mirrors with very high reflectivity in multiple wavelength bands. In addition, unlike in a linac, the electron beam in a storage ring is recycled so that it participates in the FEL interaction repeatedly over a large number of passes. Therefore, the physics challenges for the two-color operation of a storage-ring FEL include the control and management of two competing lasing processes and maintenance of simultaneous lasing at two wavelengths in multiple passes. In this paper, we report a systematic experimental study of a storage ring two-color FEL at the Duke FEL facility. Simultaneous lasing of two FEL wavelengths (IR and UV) has been realized with the use of a pair of dual-band high-reflectivity mirrors developed using a new coating technology. The research has been conducted with two different undulator configurations, showing good performance of the two-color operations in terms of wavelength tunability, power control and power stability. The degradation of FEL mirrors has also been studied in terms of its impact on the wavelength tuning and power stability. In addition, we have confirmed the simultaneousness of two-color lasing at the time scale of FEL micropulses with power modulation at a larger time scale, displaying a temporal structure of macropulses in the two-color laser beams.

\section{FEL CONFIGURATIONS AND DUAL-BAND MIRRORS}

The Duke storage ring FEL system [38] is comprised of an optical cavity and four FEL undulators [see Fig. 1(a)]. The FEL cavity is a two-mirror, near-concentric optical resonator. The cavity length is $53.7 \mathrm{~m}$, which allows the round-trip time of the trapped optical beam to be matched to the revolution time of the relativistic electron beam circulating in the storage ring (at $2.79 \mathrm{MHz}$ ). Typically, four electromagnetic undulators are installed in the $34.21 \mathrm{~m}$ long FEL straight section. To provide user research with choices of photon polarization, undulators with different polarizations are used, including two planar OK-4 undulators (OK-4B and OK-4C, 33 periods

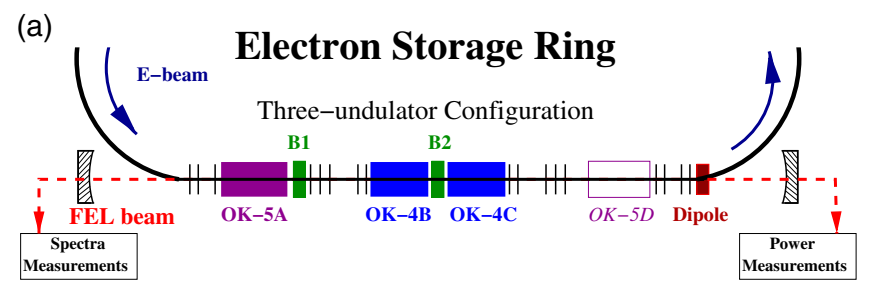

(b)
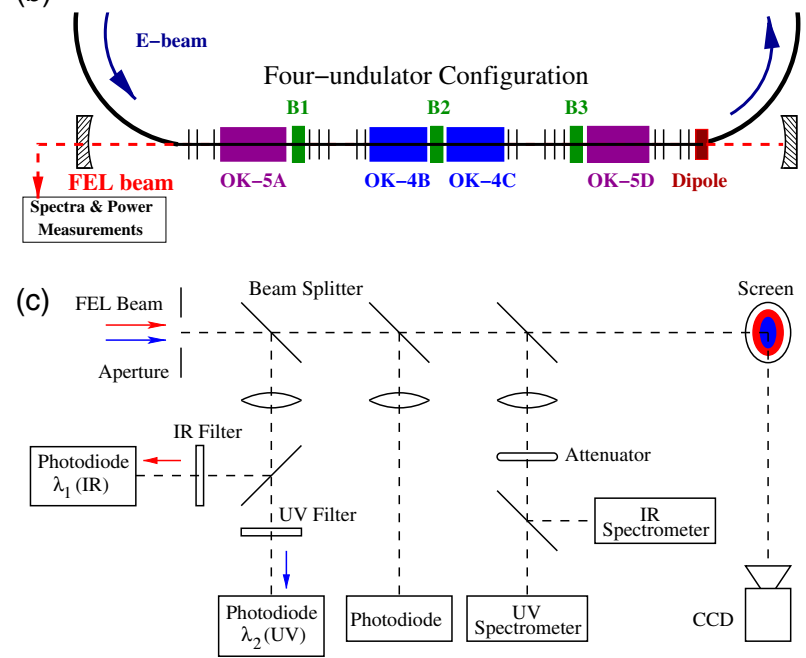

FIG. 1. Two different undulator configurations for the twocolor operation. (a) Three-undulator configuration with the helical OK-5A undulator lasing in IR and the OK-4 optical klystron lasing in UV; (b) Four-undulator configuration with two OK-5 undulators (OK-5A and OK-5D) working as an optical klystron to replace the single OK-5A undulator in (a); (c) A schematic layout of a set of typical optical diagnostics for the two-color FEL operation.

each) in the middle of the straight section and two helical OK-5 undulators outboard (OK-5A and OK-5D, 30 periods each). For this research, two undulator configurations have been used, with either three or four undulators. In the threeundulator configuration [see Fig. 1(a)], three upstream undulators are energized using two independent power supplies with the helical OK-5A undulator tuned to produce radiation at the first wavelength $\lambda_{1}$ and the planar OK-4 optical klystron (undulators OK-4B and OK-4C) at the second wavelength $\lambda_{2}$, while the downstream OK-5D undulator is turned off. In the four-undulator configuration [see Fig. 1(b)], all four undulators are energized using three power supplies so that the OK-5A and OK-5D undulators (with two power supplies to provide the same current) can be tuned to radiate at $\lambda_{1}$ and OK-4 optical klystron at $\lambda_{2}$.

By changing either the electron beam energy or undulator field strength, the FEL lasing wavelength can be tuned around the center wavelength of the undulator radiation, $\lambda_{\text {cen }}$,

$$
\lambda_{\text {cen }}=\frac{\lambda_{u}}{2 \gamma^{2}}\left(1+p \frac{K^{2}}{2}\right),
$$


where $\lambda_{u}$ is the undulator period, $\gamma=E /\left(m c^{2}\right)$ is the Lorentz parameter for an electron with energy $E$ and rest mass $m, K=e B_{0} \lambda_{u} /\left(2 \pi m c^{2}\right)$ describes the undulator strength with $B_{0}$ as the amplitude of the fundamental harmonic of undulator magnetic field, and the polarization parameter $p=1$ (or 2) for a planar (or helical) undulator, respectively. The primary wavelength control is realized by changing the OK-4 and OK-5 currents.

The FEL mirrors are produced using oxide-based multilayer dielectric coatings to achieve very high reflectivity in a particular wavelength band. Recent development in coating technology allows production of mirrors with more than one high-reflectivity band in the wavelength range from near IR to near UV. A set of dual-band FEL mirrors were specially developed for the two-color FEL research with one high-reflectivity band in the IR region around $720 \mathrm{~nm}$ and the other in the UV region around $360 \mathrm{~nm}$ [39]. These wavelength bands were chosen to explore harmonic FEL lasing with $\lambda_{1}=2 \lambda_{2}$.

The reflectivity performance of the FEL mirrors degrades when they are subjected to synchrotron radiation $[40,41]$. For the storage ring based FEL, the mirrors suffer from a number of degradation mechanisms, including two most important ones: (1) radiation induced color center formation in the coating layers [42], and (2) carbon deposition onto the top coating layer $[43,44]$. A specific set of conditioning procedures have been developed to "season" new mirrors by conditioning the mirror surface using visible undulator radiation, gradually increasing the intensity over a period of several hours. This practice helps to remove surface contaminants while reducing creation of defects in the top coating layers. Consequently, it improves mirror durability and extends useful mirror lifetime.

Figure 2 shows the measured round-trip losses in the IR and UV bands for the pair of FEL mirrors used in the study after they were subjected to different amounts of radiation exposure over a period of 25 months. The round-trip cavity loss was measured using a pass-by-pass optical cavity ringdown technique $[45,46]$. After the initial conditioning, the minimum round-trip cavity loss [loss curves L1 in Fig. 2, where 2(a) and 2(b) show two separated segments in IR and UV] was $0.15 \%$ around $704 \mathrm{~nm}$ in the IR region and $1.2 \%$ around $365 \mathrm{~nm}$ in the UV region. This set of mirrors was used occasionally for a short duration in the two-color experiments during a 24-month period, followed by the second set of cavity loss measurements (loss curves L2). These measurements showed that the minimum IR loss was increased to $0.25 \%$ and blueshifted to $695 \mathrm{~nm}$ while the minimum UV loss was decreased slightly to $0.98 \%$ and redshifted to $368 \mathrm{~nm}$. The widths of high-reflectivity regions in both IR and UV were narrowed as the result of radiation exposure. This trend continued as the mirrors were used for further study. After some additional exposure in the last month of the study, the location of the minimum IR loss was further blueshifted to $689 \mathrm{~nm}$ with the round-trip
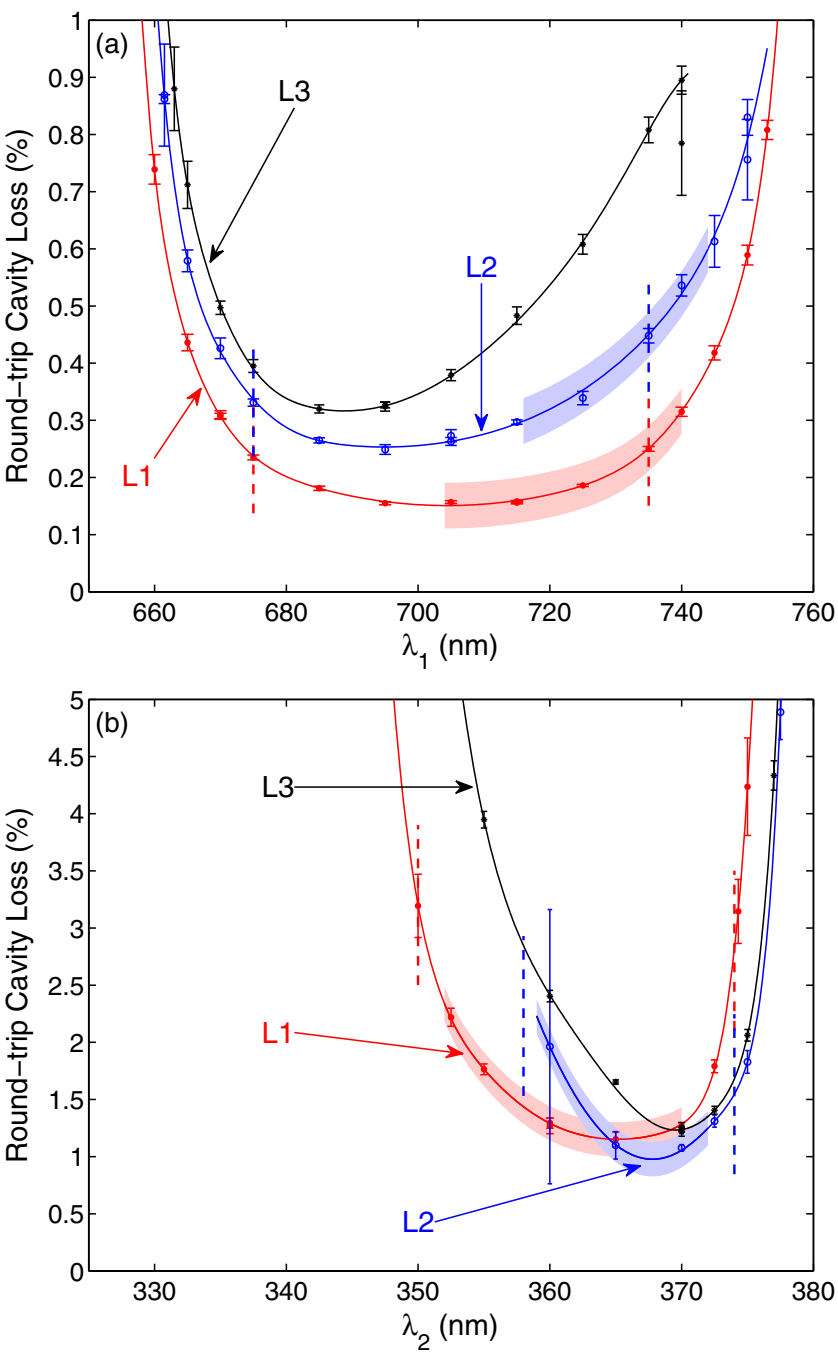

FIG. 2. Measured round-trip cavity losses around the highreflectivity bands in the (a) IR and (b) UV regions of the FEL mirrors over a 25 -month period. Loss curves L1 present the cavity losses after initial conditioning of the FEL mirrors. Loss curves L2 and L3 show the continued degradation of the mirrors' reflectivity after they have been exposed to substantial accumulated radiation, estimated to be 9.1 and 11.2 ampere-hours respectively with electron beam energy between 280 and $560 \mathrm{MeV}$. The ranges enclosed by the dashed lines represent the ranges of single wavelength tuning, and the shaded regions on the curves represent the tuning ranges of the harmonic lasing. (see Sec. III).

loss increased to $0.32 \%$, and the location of the minimum UV loss was redshifted to $370 \mathrm{~nm}$ with a loss of $1.2 \%$. In all cases, the cavity loss was dominated by radiation absorption in the dielectric coating layers, with carbon deposition onto the top layer being the major cause. The mirror transmission was very low, estimated to be $1 \times 10^{-4}$ around $720 \mathrm{~nm}$ and $4 \times 10^{-5}$ around $360 \mathrm{~nm}$ per mirror when the mirrors were relatively new, corresponding to loss curves L1 in Fig. 2.

In the experiments, the lasing processes of two colors were found to compete with each other due to the sharing of the gain medium, a single electron beam. Therefore, to 
obtain simultaneous lasing of two colors, it is critical to pair a set of undulators with a high (or low) FEL gain with a large (or small) loss band of the FEL mirrors. In our study of two-color lasing, the OK-5 system with either one or two undulators has a relatively low gain because its undulators are located either upstream or downstream of the optical cavity with a poor transverse overlap between optical and electron beams in the undulators. The lower gain of OK-5 is compensated by operating OK-5 at $\lambda_{1}$ in the IR region where the optical cavity has smaller losses (see Fig. 2). Located in the middle of the FEL cavity, two OK-4 undulators with an optical klystron configuration have a higher gain and thus they are chosen to be operated at $\lambda_{2}$ in the UV region where the cavity losses are also larger. To provide a better gain matching, the OK-4 gain at $\lambda_{2}$ needs to be further reduced by tuning buncher $B 2$ [see Fig. 1(a)] to force OK-4 to lase in the low-gain region of its gain spectrum. With the four-undulator configuration, the OK-5 gain at $\lambda_{1}$ can be increased by tuning up the OK-5 optical klystron [see Fig. 1(b)]. In addition, for both undulator configurations the relative gain at the two wavelengths can be further controlled by tuning bunchers (B1 and B2 for the three-undulator configuration; all the three bunchers for the four-undulator configuration) with small phase slippages (a few lasing wavelengths), by adjusting the FEL cavity detune i.e. the time synchronization between electron and optical beams, by changing the electron orbit in the respective undulators, and/or by tilting the cavity optical axis.

All the two-color FEL experiments reported in this paper were conducted with a single-bunch, $500 \mathrm{MeV}$ electron beam in the Duke storage ring. For the dc power measurements, the FEL was operated in the quasicontinuous-wave mode with a small rf detune. The FEL spectra at two wavelengths were measured using two spectrometers from Ocean Optics, a USB4000 spectrometer with a wavelength range of 477-1146 nm, and a HR4000 spectrometer with a wavelength range of $220-447 \mathrm{~nm}$. The relative extracted FEL power of each color was measured using a photodiode after filtering out the signal of the other color. The absolute power, such as the total extracted FEL power from one mirror, was measured using a thermal power meter. The thermal power meter was also used to calibrate the two photodiodes so that the extracted power of each color could be determined absolutely (in $\mathrm{mW}$ ). Several versions of the FEL diagnostics were developed with incremental improvements over the course of this research. Figure 1(c) shows the most commonly used version, a compact FEL diagnostic system with both spectrum measurements and power measurements installed on a common optical bench.

Two-color lasing has been studied using either threeundulator or four-undulator configuration with different levels of cavity loss. The time-consuming measurements of cavity loss were only carried out occasionally (Fig. 2), typically just before or after a major experimental run. Therefore, each run can only be matched roughly with a
TABLE I. The study of two-color FEL lasing is conducted using five experimental runs (Exp. Run) with various undulator configurations (Undulator Config.) and different levels of cavity loss in a period of 25 months.

\begin{tabular}{|c|c|c|c|}
\hline $\begin{array}{l}\text { Exp. } \\
\text { Run }\end{array}$ & $\begin{array}{l}\text { Loss } \\
\text { Curve }\end{array}$ & $\begin{array}{l}\text { Undulator } \\
\text { Config. }\end{array}$ & Comment \\
\hline Run 0 & L1 & 3-undulator & Two-color FEL exploration. \\
\hline Run 1 & L1 & 3-undulator & Results reported in Ref. [47]. \\
\hline Run 2 & L1 & 3-undulator & $\begin{array}{l}\text { dc power stability and } \\
\text { temporal structure. }\end{array}$ \\
\hline Run 3 & L2 & $\begin{array}{l}\text { 4-undulator } \\
\text { 3-undulator }\end{array}$ & Wavelength tuning. \\
\hline Run 4 & L3 & $\begin{array}{l}\text { 4-undulator } \\
\text { 3-undulator }\end{array}$ & $\begin{array}{l}\text { Power control and dc } \\
\text { power stability. }\end{array}$ \\
\hline
\end{tabular}

particular set of loss curves. Table I summarizes five main experimental runs, showing the undulator configurations used and the corresponding cavity loss curves around the time of the study. The earlier results of two-color FEL lasing using the three-undulator configuration have been published in Ref. [47]. In this paper, we mainly report the new experimental results in either three- or four-undulator configuration. When appropriate, these results are compared with those reported in Ref. [47].

\section{WAVELENGTH TUNING FOR TWO-COLOR LASING}

With this two-color FEL, the lasing wavelengths can be tuned in multiple ways. For example, tuning one wavelength while keeping the other fixed can be achieved by varying the current(s) of the respective undulator(s) and simultaneously tuning bunchers for wavelength fine adjustments. Also, the two harmonically related high-reflectivity bands of the FEL mirrors allow the wavelength tuning of harmonic lasing where the two wavelengths are tuned simultaneously with the UV lasing locked to the second harmonic of the IR lasing $\left(\lambda_{1} \simeq 2 \lambda_{2}\right)$. During the tuning process, the FEL gains of both colors have to be large enough to overcome their respective cavity losses and to keep the net gains (FEL gains minus losses) reasonably matched at two lasing wavelengths. Consequently, the wavelength tuning ranges of two-color lasing are determined by two main factors: (1) the available FEL gains and (2) the high-reflectivity bands of the FEL mirrors. The FEL gain of each color depends on the number of undulators used, the level of the electron beam current and the tuning of various knobs for gain control. For a given FEL gain, the wavelength tuning range for each color is limited by the width of the high-reflectivity spectral region around the minimum round-trip cavity loss. Especially for the harmonic two-color lasing, the tuning range is determined by the amount of overlap of the two high-reflectivity wavelength bands.

Figure 3 shows the results of single wavelength tuning using the four-undulator configuration with loss curves L2. 

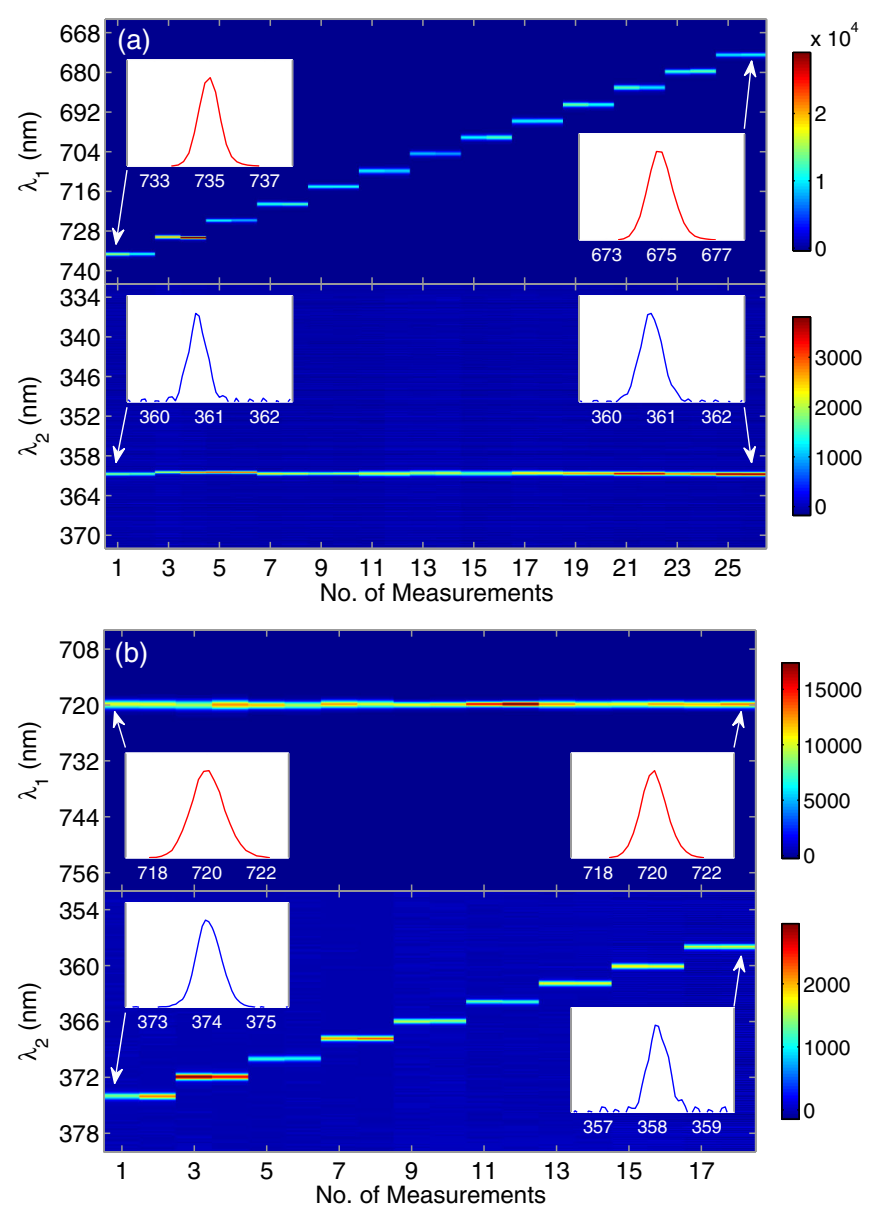

FIG. 3. Tuning of the (a) IR and (b) UV wavelength of the twocolor lasing with the four-undulator configuration and loss curves L2 (Run 3). In (a), the IR lasing $\left(\lambda_{1}\right)$ is shown to be tuned from $734.97 \mathrm{~nm}$ to $674.93 \mathrm{~nm}\left(\Delta \lambda_{1}=60.04 \mathrm{~nm}\right)$ while fixing the UV lasing $\left(\lambda_{2}\right)$ around $360.69 \pm 0.08 \mathrm{~nm}$. In (b), $\lambda_{2}$ is tuned from $374.02 \mathrm{~nm}$ to $358.10 \mathrm{~nm}\left(\Delta \lambda_{2}=15.92 \mathrm{~nm}\right)$ while keeping $\lambda_{1}$ around $720.06 \pm 0.07 \mathrm{~nm}$. The beam current is maintained between $12.03 \mathrm{~mA}$ and $12.78 \mathrm{~mA}$ in both (a) and (b).

In Fig. 3(a), $\lambda_{1}$ is tuned by varying the magnetic field of the OK-5 undulators and the settings of bunchers B1 and B3, while $\lambda_{2}$ is kept nearly constant by slightly tuning the magnetic field of the OK-4 undulators and buncher B2. Figure 3(b) presents the tuning of UV wavelength, where $\lambda_{2}$ is tuned by adjusting OK-4 magnetic field strength and the setting of buncher B2. Since B2 is a shared buncher by the OK-4 and OK-5 optical klystrons, bunchers B1 and B3 as well as the OK-5 undulators are tuned to compensate for any minor wavelength shift in $\lambda_{1}$.

To demonstrate the wavelength tuning of harmonic two-color lasing, the magnetic field strengths of OK-4 and OK-5 undulators as well as bunchers were varied simultaneously so that the UV lasing was locked to the second harmonic of the IR lasing $\left(\lambda_{1} \simeq 2 \lambda_{2}\right)$. Figure 4 shows eight harmonically related pairs of spectra (normalized to their respective peak intensity) where the tuning

\begin{tabular}{|c|c|}
\hline$\left(\lambda_{1}, \mathrm{~K}_{\mathrm{OK}-5}\right)=(744.08 \mathrm{~nm}, 3.47)$ & $\left(\lambda_{2}, \mathrm{~K}_{\mathrm{OK}-4}\right)=(371.99 \mathrm{~nm}, 3.47)$ \\
\hline (739.88 nm, 3.43) & $(370.13 \mathrm{~nm}, 3.47)$ \\
\hline$(735.96 \mathrm{~nm}, 3.41)$ & (368.07 nm, 3.45) \\
\hline (732.06 nm, 3.38) & (366.06 nm, 3.44) \\
\hline$(728.02 \mathrm{~nm}, 3.36)$ & (364.06 nm, 3.42) \\
\hline (723.89 nm, 3.32) & (361.98 nm, 3.41) \\
\hline (719.83 nm, 3.35) & (359.96 nm, 3.38) \\
\hline (716.15 nm, 3.34) & (358.25 nm, 3.36) \\
\hline $\begin{array}{c}716720724728732736740744 \\
\lambda_{1}(\mathrm{~nm})\end{array}$ & $\begin{array}{r}3583603623643663 \\
\lambda_{2}(\mathrm{~nm})\end{array}$ \\
\hline
\end{tabular}

FIG. 4. Wavelength tuning with the UV wavelength set to the second harmonic of the IR wavelength using the four-undulator configuration and loss curves L2. $\lambda_{1}$ is tuned from $716.15 \mathrm{~nm}$ to $744.08 \mathrm{~nm}$ and $\lambda_{2}$ is varied from $358.25 \mathrm{~nm}$ to $371.99 \mathrm{~nm}$ simultaneously. During the measurement, the beam current was maintained between $12.35 \mathrm{~mA}$ and $12.94 \mathrm{~mA}$.

ranges of $\Delta \lambda_{1}=27.93 \mathrm{~nm}$ and $\Delta \lambda_{2}=13.74 \mathrm{~nm}$ are achieved. It should be noticed that for the two pairs of spectra with UV wavelength at $358.25 \mathrm{~nm}$ and $359.96 \mathrm{~nm}$, the signal-to-noise ratio of the UV spectra is much lower than that of the other UV spectra, indicating that this spectral region is close to the short wavelength limit of UV lasing.

Table II summarizes the demonstrated wavelength tuning ranges in two experimental runs, Run 1 and Run 3. The same tuning range $\left(\Delta \lambda_{1} \simeq 60 \mathrm{~nm}\right)$ of the IR single wavelength tuning was achieved in both runs. The increased cavity loss in the IR band of the degraded FEL mirrors [L2 in Fig. 2(a)] was compensated by a substantial increase of the IR FEL gain in Run 3 by using the OK-5 optical klystron with both OK-5A and OK-5D undulators. In addition, the beam current was lowered to about $12 \mathrm{~mA}$ in Run 3 (compared to $16 \mathrm{~mA}$ in Run 1) to reduce OK-5 gain so that the same IR tuning range $\left(\Delta \lambda_{1} \simeq 60 \mathrm{~nm}\right)$ as in Run 1 could be reproduced. On the other hand, the tuning range of the UV single wavelength tuning in Run 3 $\left(\Delta \lambda_{2} \simeq 16 \mathrm{~nm}\right)$ was narrowed compared to that in Run 1 $\left(\Delta \lambda_{2} \simeq 24 \mathrm{~nm}\right)$. Especially, the short wavelength limit of the tuning range was redshifted by about $8 \mathrm{~nm}$. This redshift and the shrinkage of the tuning range were consistent with the measured bandwidth narrowing of the UV loss curve shown in Fig. 2(b). Further, the tuning range of harmonic two-color lasing is determined by the amount of overlap of the low-loss regions of the highreflectivity wavelength bands in IR and UV. In fact, the tuning range of the harmonic lasing was narrowed due to a dramatic increase of the loss in the UV band below $360 \mathrm{~nm}$ [Fig. 2(b)]. 
TABLE II. Summary and comparison of wavelength tuning ranges in two experimental runs with different undulator configurations, loss curves and beam currents. In Run 1, the threeundulator configuration and the relatively fresh FEL mirrors (loss curves L1) were used with the beam current $\sim 16 \mathrm{~mA}$ for the single wavelength tuning and $\sim 20 \mathrm{~mA}$ for the harmonic lasing tuning [47]. In Run 3, four undulators were employed with the usage of the degraded FEL mirrors (loss curves L2) and the beam current $\sim 12 \mathrm{~mA}$ for all the three sets of wavelength tuning measurements. The tuning ranges are shown in Fig. 2 with those of the single wavelength tuning enclosed by the dashed lines and those of the harmonic lasing represented by the shaded regions.

\begin{tabular}{lcc}
\hline \hline & Run 1 & Run 3 \\
Wavelength & & \\
Tuning & 3-undulator, L1 & 4-undulator, L2 \\
\hline IR $\left(\lambda_{1}\right)(\mathrm{nm})$ & $674.92-734.88$ & $674.93-734.97$ \\
$\Delta \lambda_{1}(\mathrm{~nm})$ & 59.96 & 60.04 \\
Fixed $\lambda_{2}(\mathrm{~nm})$ & 360.05 & 360.69 \\
UV $\left(\lambda_{2}\right)(\mathrm{nm})$ & $349.93-373.96$ & $358.10-374.02$ \\
$\Delta \lambda_{2}(\mathrm{~nm})$ & 24.03 & 15.92 \\
Fixed $\lambda_{1}(\mathrm{~nm})$ & 720.00 & 720.06 \\
IR and UV & & \\
$\lambda_{1}(\mathrm{~nm})$ & $703.88-740.03$ & $716.15-744.08$ \\
$\lambda_{2}(\mathrm{~nm})$ & $351.98-370.02$ & $358.25-371.99$ \\
$\Delta \lambda_{1}(\mathrm{~nm})$ & 36.15 & 27.93 \\
$\Delta \lambda_{2}(\mathrm{~nm})$ & 18.04 & 13.74 \\
\hline \hline
\end{tabular}

Interesting observations can be made by studying the lasing spectral widths. For the storage ring FEL lasing at a single wavelength, the lasing spectral width depends on a number of accelerator setup and operational conditions, as well as the electron beam parameters. A few important ones include the FEL undulator configuration, the cavity loss, tuning of the optical klystron, setting of the rf detune, alignment of the optical axis and the electron beam orbit in the undulators, as well as the electron beam energy and current. For a single-color FEL operated in the small-signal, small-gain region, under certain operational conditions, the relative spectral width of FEL lasing is proportional to that of the spontaneous radiation spectrum $[48,49]$ i.e. $\sigma_{\lambda} / \lambda \propto 1 / N_{\text {un }}$ for a single undulator and $\sigma_{\lambda} / \lambda \propto$ $1 /\left[2\left(N_{\text {un }}+N_{B}\right)\right]$ for an optical klystron, where $N_{\text {un }}$ is the number of periods of one undulator, and $N_{B}$, representing the relative phase delay between the laser and electron beams, is much smaller than $N_{\text {un }}$ in our case. In our study of two-color lasing, the FEL is operated in the quasicontinuous-wave mode, in which the spontaneous radiation spectra are substantially broadened due to the relatively large electron beam energy spread induced by FEL interactions. However, for this two-color FEL, the relative spectral width $\left(\sigma_{\lambda} / \lambda\right)$ is still found to be related to the number of undulator periods used.

The spectral widths of harmonic two-color lasing with wavelength tuning have been analyzed for Run 1 and Run 3, and the results are shown in Fig. 5. In Run 1 with the

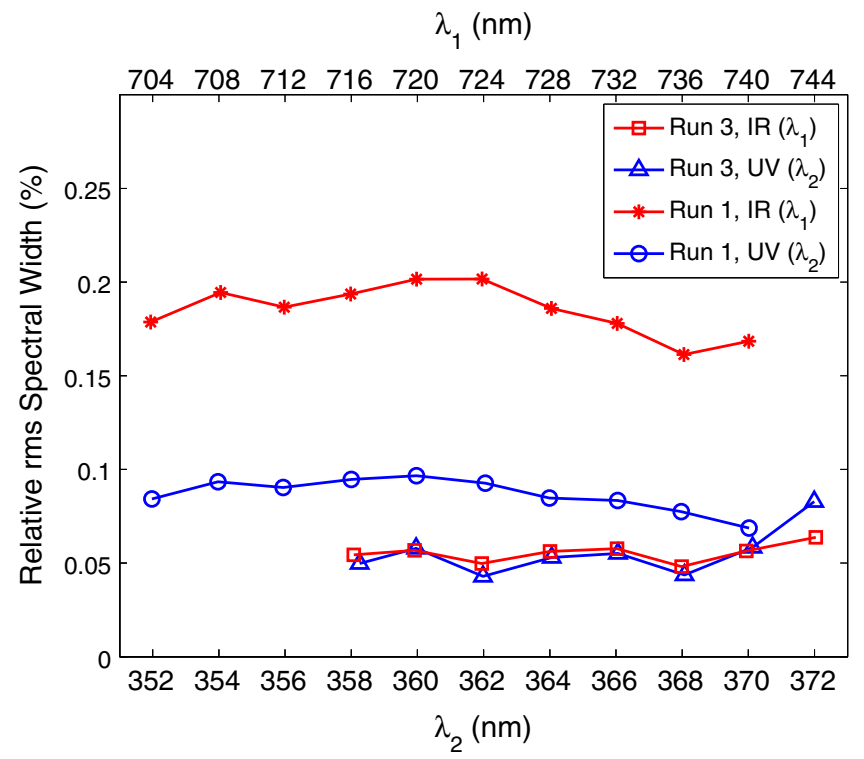

FIG. 5. Relative spectral widths (rms) in the wavelength tuning of the harmonic lasing in two runs, Run 1 and Run 3. Run 1 curves (starred and circled) represent the relative spectral widths in the measurement shown in Fig. 3 of Ref. [47]. In this measurement, the three-undulator configuration was used with loss curves L1 and beam current about $20 \mathrm{~mA}$. Run 3 curves (squared and triangled) show the relative spectral widths in Fig. 4, where four undulators are used with loss curves L2, and the beam current is set to about $12 \mathrm{~mA}$.

three-undulator configuration (OK-5A undulator with about 30 periods and OK-4 optical klystron with about 66 periods), $\sigma_{\lambda_{1}} / \lambda_{1}$ (starred) was larger than $\sigma_{\lambda_{2}} / \lambda_{2}$ (circled) roughly by a factor of 2 . In Run 3, OK-5D undulator was turned on to form the OK-5 optical klystron. Because OK-5D undulator was located about $20 \mathrm{~m}$ downstream from OK-5A undulator, separated by a number of quadrupoles and the pair of OK-4 undulators [see Fig. 1(b)], the OK-5 optical klystron was not expected to perform as effectively as the OK-4 optical klystron with two adjacent undulators. Nevertheless, we found a significant reduction of the IR spectral bandwidth using the OK-5 optical klystron in Run 3, reducing $\sigma_{\lambda_{1}} / \lambda_{1}$ from $(1.9 \pm 0.1) \times$ $10^{-3}$ (Run 1) to $(5.5 \pm 0.5) \times 10^{-4}$ (Run 3). Comparing the UV lasing in these two runs for the common wavelength tuning range from about 358 to about $370 \mathrm{~nm}, \sigma_{\lambda_{2}} / \lambda_{2}$ (circled) in Run 1 were slightly larger than that (triangled) in Run 3. This may be the result of having a higher net gain in Run 1 due to a larger beam current (about $20 \mathrm{~mA}$ in Run 1 vs about $12 \mathrm{~mA}$ in Run 3) and lower cavity loss in this wavelength range [loss curves L1 vs L2 in Fig. 2(b)].

\section{POWER CONTROL}

The results of the spectral measurements in the previous section showing two-color lasing with wavelength tunability have demonstrated the capability of providing 
effective gain balance for two lasing processes. In this section, the precise power control of two-color lasing as well as the stability of the lasing power are presented. In an oscillator FEL, there are three different power parameters which are important to describe FEL operation. The first one is the direct FEL power $\left(P_{\mathrm{FEL}}\right)$, the coherent power emitted by the electron beam in the FEL interaction. The second power parameter is the intracavity power $\left(P_{\text {intra }}\right)$, the amount of the laser power trapped inside the laser cavity. The third one is the extracted FEL power $P_{\text {extract }}$ (from one of the FEL mirrors). In the study of an oscillator FEL, the direct FEL power is particularly important, while only the extracted FEL power can be directly measured. In order to relate the extracted FEL power to the direct FEL power, a technique based upon the measured electron beam bunch length using an image dissector tube [50] was developed. This technique is particularly useful for crosscalibrating the photodiodes used to measure the extracted FEL power at each wavelength of two-color lasing. With proper cross-calibration, the study of the FEL power is based upon the measured extracted FEL power from the upstream FEL mirror.

The direct FEL power $P_{\text {FEL }}$ can be written as [51-56]

$$
P_{\mathrm{FEL}} \approx \alpha P_{\mathrm{syn}} \frac{\sigma_{E, \mathrm{FEL}}^{2}-\sigma_{E, 0}^{2}}{\sigma_{E, \mathrm{FEL}}}
$$

where $\alpha$ is a numerical factor depending on the undulator configuration and FEL operational conditions; $P_{\text {syn }}$ is the total synchrotron radiation power emitted by the electron bunch in the entire storage ring; $\sigma_{E, \text { FEL }}$ and $\sigma_{E, 0}$ are the rms relative electron beam energy spread with FEL turned on and off, respectively. In the microwave instability region with large enough bunch current and/or with FEL lasing to increase the electron beam energy spread, the electron beam energy spread and bunch length are found to be related in a simple manner due to the longitudinal phase space rotation [55]:

$$
\sigma_{E} \simeq k \sigma_{b},
$$

where $\sigma_{E}$ and $\sigma_{b}$ are the relative energy spread (rms) and the bunch length (rms) of the electron beam, respectively; $k=\omega_{s} /\left(\alpha_{c} c\right)$ is a numerical factor related to the frequency of synchrotron oscillation $\omega_{s}$ and the momentum compaction factor $\alpha_{c}$. Since the beam current (a few mA to tens of $\mathrm{mA}$ ) used in a typical FEL operation at the Duke storage ring is significantly beyond the microwave instability threshold [57], the electron beam energy spreads $\left(\sigma_{\mathrm{FEL}}\right.$ and $\sigma_{0}$ ) can be experimentally determined by measuring the electron beam bunch length and consequently the direct FEL power can be rewritten in terms of the measured bunch length as

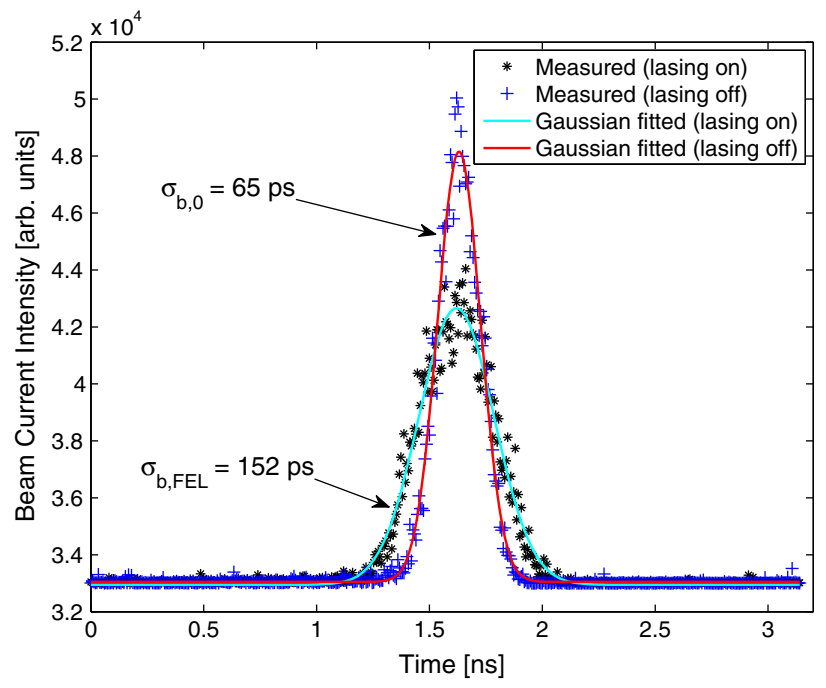

FIG. 6. Electron beam bunch length measurements in the twocolor operation with the four-undulator configuration and loss curves L2 (Run 3). The data points marked by "star" and "plus" represent the measured longitudinal profiles of the electron bunch with the lasing turned on and off, respectively. The data are fitted using Gaussian distribution (solid curves). The measured dissector resolution $\sigma_{b \text {,res }}=69 \mathrm{ps}$. The FEL is operated at $\lambda_{1}=$ $712.31 \mathrm{~nm}$ and $\lambda_{2}=367.01 \mathrm{~nm}$. The beam current is $12.34 \mathrm{~mA}$.

$$
P_{\mathrm{FEL}} \approx \alpha^{\prime} P_{\mathrm{syn}} \frac{\sigma_{b, \mathrm{FEL}}^{2}-\sigma_{b, 0}^{2}}{\sigma_{b, \mathrm{FEL}}}
$$

where $\sigma_{b, \mathrm{FEL}}$ and $\sigma_{b, 0}$ are the measured bunch lengths with FEL turned on and off, respectively, and $\alpha^{\prime}$ is a numerical factor. Figure 6 shows two measured longitudinal profiles of the electron bunch in Run 3 with the lasing turned on and off respectively. Compared to the case when the FEL is turned off with $\sigma_{b, 0}=65 \mathrm{ps}$, the bunch is significantly lengthened to $\sigma_{b \text {,FEL }}=152 \mathrm{ps}$ due to the FEL induced energy spread when FEL is turned on.

To represent the direct FEL power using the measurable extracted FEL power, the extracted power $\left(P_{\text {extract }}\right)$ can be first related to the intracavity power $\left(P_{\text {intra }}\right)$ as

$$
P_{\text {extract }}-P_{0}=T_{\text {mirror }} P_{\text {intra }},
$$

where $T_{\text {mirror }}$ is the transmission factor of the FEL mirror (upstream); $P_{0}$ is the residual extracted power when the lasing is turned off, which represents the trapped broadband spontaneous undulator radiation inside the FEL cavity. This power offset $P_{0}$ can be experimentally determined by turning the FEL off with a large rf detune. In the steadystate FEL operation, the direct FEL power $\left(P_{\mathrm{FEL}}\right)$ balances the round-trip cavity loss and thus the intracavity power can be written in terms of the direct FEL power as

$$
P_{\text {intra }}=\frac{P_{\mathrm{FEL}}}{l_{\text {cavity }}},
$$


where $l_{\text {cavity }}$ is the relative round-trip cavity loss. Consequently, the corrected extracted FEL power (after subtracting the power offset $P_{0}$ ) and the direct FEL power can be related as

$$
P_{\text {extract }}^{\prime}=A P_{\mathrm{FEL}}
$$

where $P_{\text {extract }}^{\prime}=P_{\text {extract }}-P_{0}$, and $A=T_{\text {mirror }} / l_{\text {cavity }}$.

For the FEL operations at Duke, the extracted FEL power through either FEL mirror can be directly measured using photodiodes, while the direct FEL power is determined using the measured bunch length [see Eq. (4)]. As a result, the measured extracted FEL power by a photodiode (i) can be written as

$$
P_{\mathrm{PD}, i}=C_{i} \cdot B \cdot \frac{\sigma_{b, \mathrm{FEL}}^{2}-\sigma_{b, 0}^{2}}{\sigma_{b, \mathrm{FEL}}}, \quad i=1,2, \ldots
$$

where $C_{i}$ represents the overall effects of the sensitivity of the photodiode, the transmission factor of the FEL mirror and the optics used, and the round-trip cavity loss at a particular wavelength. Therefore $C_{i}$ varies for different photodiodes. $B=\alpha^{\prime} P_{\text {syn }}$, is a common numerical factor for all the photodiodes used, related to the accelerator operational conditions. For each photodiode $(i)$, the slope $\left(k_{i}\right)$ of the linear relation $P_{\mathrm{PD}, i} \sim\left(\sigma_{b, \mathrm{FEL}}^{2}-\sigma_{b, 0}^{2}\right) / \sigma_{b, \mathrm{FEL}}$ can be determined using the measured extracted FEL power at different bunch lengths. Therefore, the cross calibration, for example, for two photodiodes $(i=1,2)$, can be achieved:

$$
\frac{C_{2}}{C_{1}}=\frac{k_{2}}{k_{1}} .
$$

Using the calibrated photodiodes, the measured extracted FEL power with different photodiodes can be used to compare the direct FEL power at different wavelengths and allow us to study the relative power partitioning of two-color lasing.

For a storage ring FEL with optical klystron configuration, the FEL power can be manipulated by adjusting the buncher setting $N_{B}$, the relative optical phase slippage between the laser and electron beams. In the four-undulator configuration in which $\mathrm{OK}-5 \mathrm{~A}$ and $\mathrm{OK}-5 \mathrm{D}$ undulators form an optical klystron sandwiching two OK-4 undulators, buncher B1 or B3 can be used as a knob to control the FEL power partitioning of two colors without causing a significant shift of the OK-4 lasing wavelength $\left(\lambda_{2}\right)$. An experimental demonstration of two-color power control by tuning B3 is shown in Fig. 7, where the measured extracted power at two wavelengths are first linearly scaled to $12 \mathrm{~mA}$ and then cross-calibrated using Eq. (9). As shown in Fig. 7, power modulation close to $100 \%$ for IR and UV was achieved, while the total FEL power was maintained relatively constant within $6.5 \%$ (rms). In the inset of Fig. 7(b), $N_{B 3}$ at the IR power minimums is linearly fitted
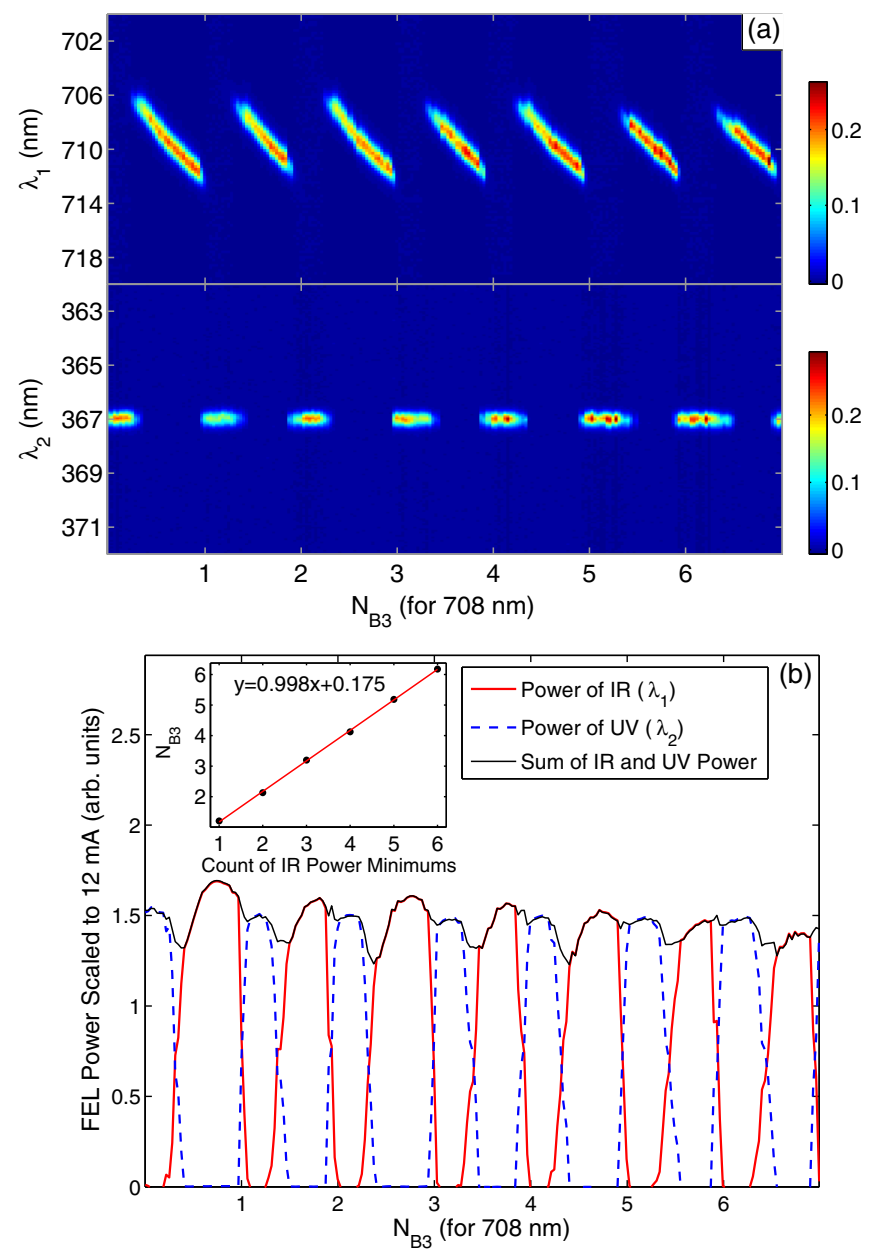

FIG. 7. Two-color FEL power control by tuning buncher B3 with the four-undulator configuration and loss curves L2. The beam current is kept between $12.01 \mathrm{~mA}$ and $12.79 \mathrm{~mA}$. (a) The measured spectra. (b) The FEL power of IR lasing (red solid), UV lasing (blue dashed), and the sum of the two powers (black).

vs the count of the IR power minimums. A modulation periodicity is clearly revealed by the fitted slope of 0.998 . The periodic power modulation in this measurement can be attributed to the gain modulation mechanism of an optical klystron, where, by tuning $N_{B 3}$, the IR beams emitted in OK-5A and OK-5D produce constructive and destructive inteference alternately for electrons of a certain energy. As shown in the previous sections, the bunchers are used not only to balance the gains of two colors but as an auxiliary knob for wavelength fine adjustments. Therefore, accompanying the power modulation, the wavelength of IR lasing was observed to shift periodically around the mean value $(709.29 \mathrm{~nm})$ by about $6.43 \mathrm{~nm}(0.9 \%)$, while the wavelength of UV lasing was maintained rather constant at $367.02 \pm 0.03 \mathrm{~nm}$.

In the three-undulator configuration, buncher B1 is also found to be useful to control the partitioning of the twocolor FEL power. This indicates that there exists one or more radiation sources downstream the OK-5A undulator, 

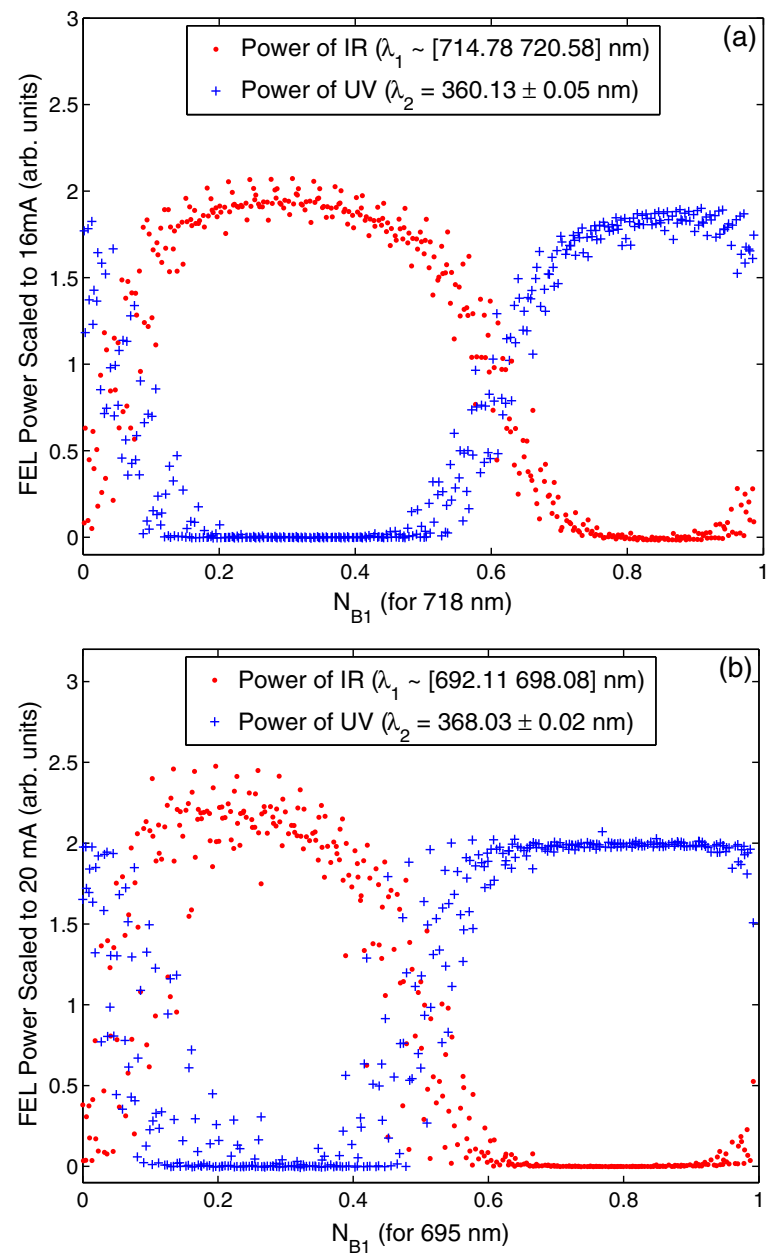

FIG. 8. Two-color FEL power control at two levels of cavity loss by tuning buncher B1 with the three-undulator configuration. (a) A revisualization of the data shown in Fig. 4(b) of Ref. [47] (loss curves L1, Run 1). $N_{B 1}$ is tuned from 0 to 11.3. All data points are projected into $\Delta N_{B 1} \sim[0,1] . \lambda_{1}$ varies between $714.78 \mathrm{~nm}$ and $720.58 \mathrm{~nm}$, while $\lambda_{2}$ is fixed at $360.13 \pm$ $0.05 \mathrm{~nm}$. The beam current is maintained between $15.60 \mathrm{~mA}$ and $16.41 \mathrm{~mA}$. (b) At a larger cavity loss (loss curves L3, Run 4). $N_{B 1}$ is varied from 0 to $11.2 . \lambda_{2}$ is fixed at $368.03 \pm 0.02 \mathrm{~nm}$ and $\lambda_{1}$ is shifted between $692.11 \mathrm{~nm}$ and $698.08 \mathrm{~nm}$. The beam current is kept between $20.01 \mathrm{~mA}$ and $20.93 \mathrm{~mA}$.

which together with OK-5A and buncher B1, form a virtual OK-5 optical klystron. Periodic power modulation similar to the one shown in Fig. 7 was realized by tuning buncher B1 in two runs of experiments with the same undulator configuration but different levels of cavity loss. Using the periodicity [the slope 0.990 for Fig. 8(a) and 0.995 for Fig. 8(b)], all the power data can be projected into the first period. In Fig. 8(a), the tuning of $N_{B 1}$ produces repeated power modulation about eleven times. The power modulation indicates a good reproducibility throughout the eleven periods. Figure 8(b) shows the power tuning using buncher B1 with larger cavity losses (L3). Comparing two cases, the cavity losses at the UV lasing wavelengths [around $360 \mathrm{~nm}$ for Fig. 8(a) and around $368 \mathrm{~nm}$ for
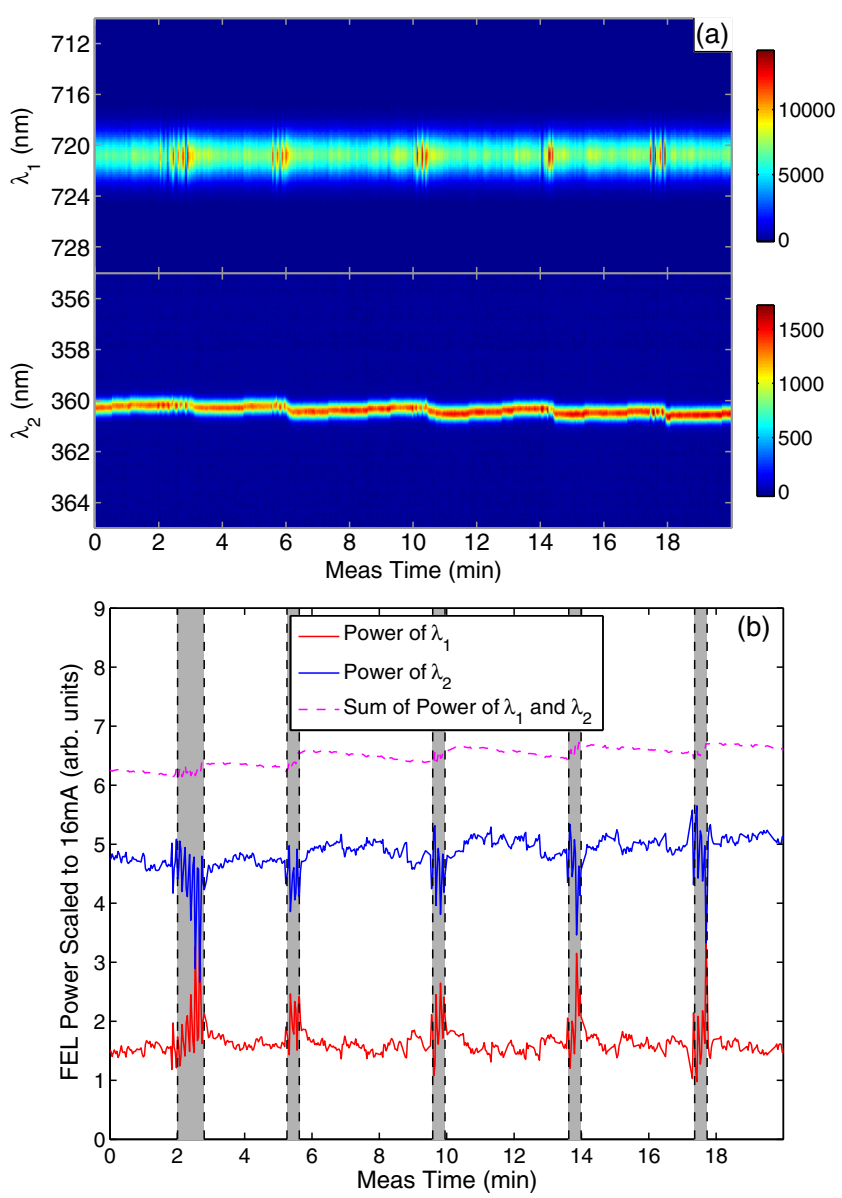

FIG. 9. Long-term FEL power measurements with the threeundulator configuration and loss curves L1 (Run 2). (a) Spectra and (b) FEL powers of two-color lasing. The narrow shaded regions in (b) represent the injection periods in which the data points are eliminated in the calculation of rms power variations. The beam current is maintained between $15.78 \mathrm{~mA}$ and $16.54 \mathrm{~mA}$ with top-off injection.

Fig. 8(b)] are similar, whereas the cavity loss at the IR lasing wavelengths [around $718 \mathrm{~nm}$ for Fig. 8(a) and around $695 \mathrm{~nm}$ for Fig. 8(b)] is twice higher in Fig. 8(b) than in Fig. 8(a). It is also found that the ratio of the IR maximum power to the UV maximum power is similar in both cases [about 1.06 and 1.07 for Fig. 8(a) and Fig. 8(b) respectively], showing that a good power balance of two-color lasing has been achieved in both cases.

The power stability of a storage ring FEL is related to many factors, including the stability of the optical axis due to the movement of FEL mirrors and the variation of the FEL cavity detune due to the temperature change. The power stability of the two-color FEL operation was examined by tracking the FEL powers at two wavelengths for a relatively long period of time. In order to maintain the power stability to a high degree, bunchers, FEL mirrors and rf detune were adjusted as needed. Figure 9 shows a selected 20-minute measurement where the powers are maintained such that the UV power is roughly three times 


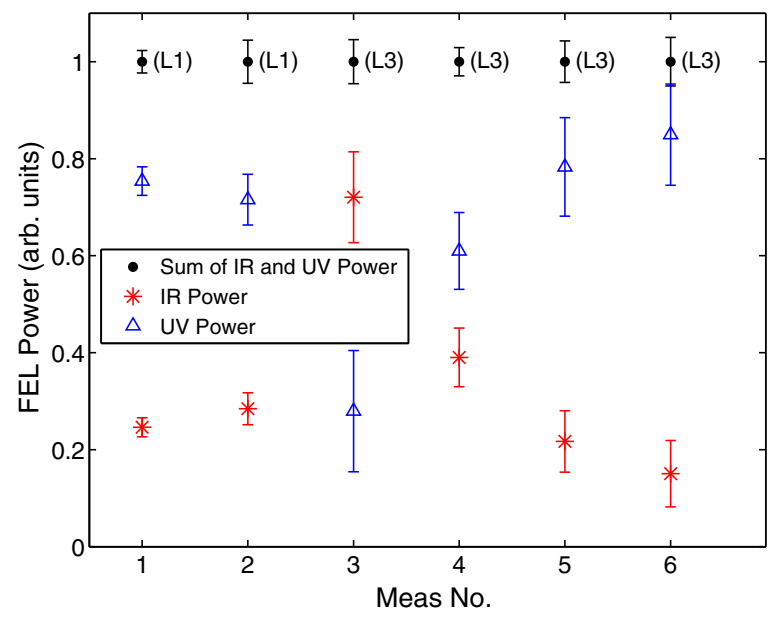

FIG. 10. Long-term FEL power measurements with the threeundulator configuration but different cavity losses. The data points and error bars represent the mean values and the rms variations of FEL power for the six 20-min measurements. The powers of each measurement are normalized to its sum of IR and UV power. Measurements no. 1 and no. 2 were taken with loss curves L1 and measurements no. 3 to no. 6 were taken with loss curves L3.

IR power with $5.2 \%$ and $11 \%$ rms variations for UV and IR, respectively (calculated after removing the data points taken during injections). In this measurement, $N_{B 2}$ was tuned as the only knob by $1.9 \%$. It can be seen from Fig. 9(b) that the power variation of one color was always compensated by that of the other color so that the total FEL power remained steady to a higher degree $(2.3 \% \mathrm{rms})$.

Figure 10 shows six 20-minute measurements with the three-undulator configuration but different levels of cavity loss. As shown in Fig. 10, the larger IR and UV power variations (shown by the rms error bars) of the measurements with the degraded FEL mirrors (measurements no. 3 to no. 6) compared to those with fresher FEL mirrors (measurements no. 1 and no. 2) indicates an increased power instability of the two-color lasing. In addition to an observed positive correlation between the mirror degradation and the instability of lasing power, temperature change may also contribute to power instability. Measurements no. 1 and no. 2 were taken with a relatively stable temperature as permitted by the weather conditions, while the rest of the measurements were taken during hot weather with more rapid temperature changes. On the other hand, the stability of the total FEL power is kept at a similar stability level for all the measurements due to the mutual coupling of two lasing processes.

\section{TWO-COLOR LASING PHASE SPACE WITH MULTIPLE TUNING KNOBS}

We focus on the investigation of the quasicontinuouswave operation of the multipass storage ring oscillator FEL. The FEL beam is developed over a large number of
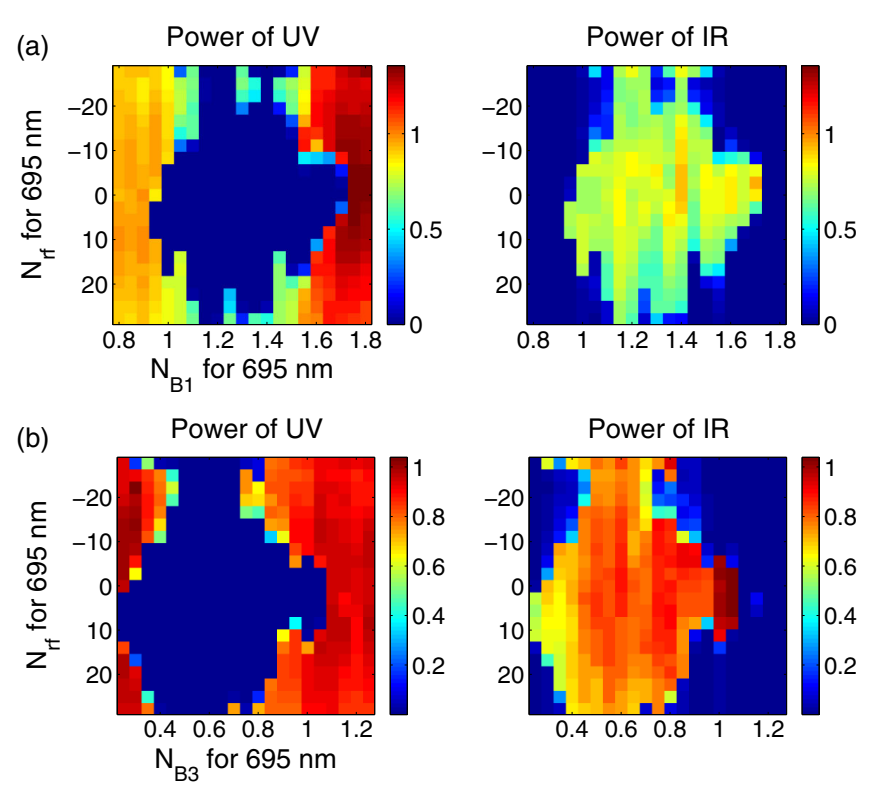

FIG. 11. Two-color lasing power in phace space with the fourundulator configuration and loss curves L3. $N_{\text {rf }}$ (y-axis) is the rf detune expressed in length normalized to $\lambda_{1}=695 \mathrm{~nm}$. (a) $N_{\mathrm{rf}} \mathrm{vs}$ $N_{B 1}$, while fixing B3 setting $\left(N_{B 3}=0.60\right.$ for $\left.695 \mathrm{~nm}\right)$. The mean wavelength of IR and UV lasings are $697.52 \mathrm{~nm}$ and $368.40 \mathrm{~nm}$, respectively. (b) $N_{\text {rf }}$ vs $N_{B 3}$, while fixing B1 setting $\left(N_{B 1}=0.25\right.$ for $695 \mathrm{~nm}$ ). The mean wavelength of IR and UV lasing are $694.32 \mathrm{~nm}$ and $367.97 \mathrm{~nm}$, respectively. In both cases, the rf detune is scanned from $-0.5 \mathrm{~Hz}\left(N_{\mathrm{rf}}=-27.69\right)$ to $0.5 \mathrm{~Hz}$ $\left(N_{\text {rf }}=27.69\right) . K_{\mathrm{OK}-4}=3.45, K_{\mathrm{OK}-5}=3.26$ and $N_{B 2}=0.77$ for $368 \mathrm{~nm}$. The beam current is maintained between $12.3 \mathrm{~mA}$ and $13.0 \mathrm{~mA}$.

interactions between the photon and electron beams inside the optical cavity, which in turn allows several means to control and manipulate the FEL processes. During each interaction, the level of electron beam microbunching can be manipulated using the buncher magnets by delaying the electrons relative to the photon beam by tens of attoseconds to a few femtoseconds, corresponding to a length scale of a fraction of the lasing wavelength to several wavelengths. Over many interactions, the synchronization condition between the FEL and electron beams can be controlled using the rf detune in a much larger time scale of a few to hundreds of femtoseconds, corresponding to a few to hundreds of lasing wavelengths. Controlling these two drastically different time/length scales can provide new insights into the two-color lasing processes. In this section, we describe a study of the two-color lasing powers as a function of the buncher setting $\left(N_{B i}, i=1,2,3\right.$; short time scale) and rf detune ( $\Delta f_{\text {rf }}$; long time scale) by visualizing the two-color lasing using two-dimensional phase space plots (see Fig. 11 and Fig. 12).

It is found that for a particular buncher setting, there exists a region of $\Delta f_{\text {rf }}$ centered at the full synchronization $\left(\Delta f_{\mathrm{rf}}=0\right)$, with the simultaneous two-color lasing forming a boundary separating IR single-color lasing (inside) and UV 
(a)

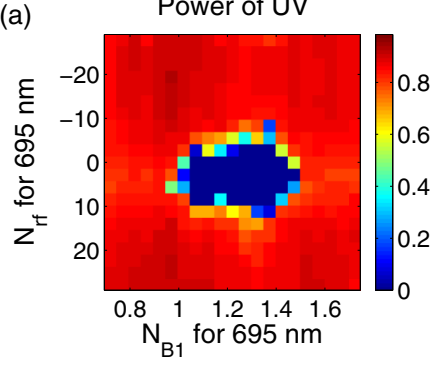

(b)

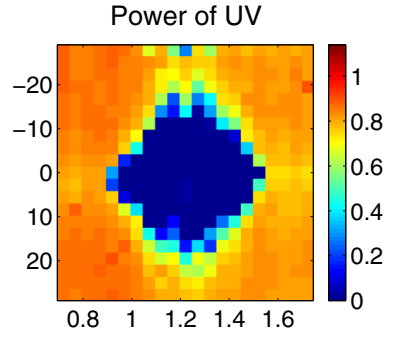

(c)

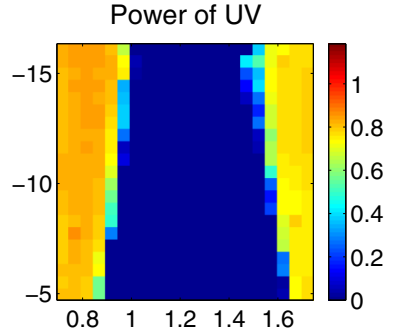

(d)

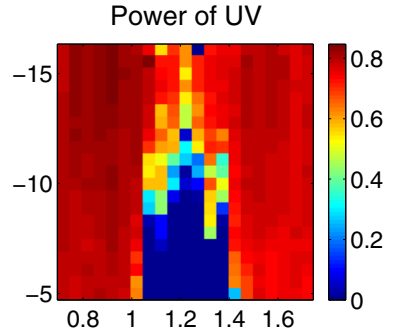

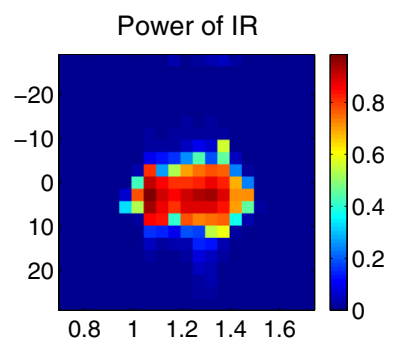
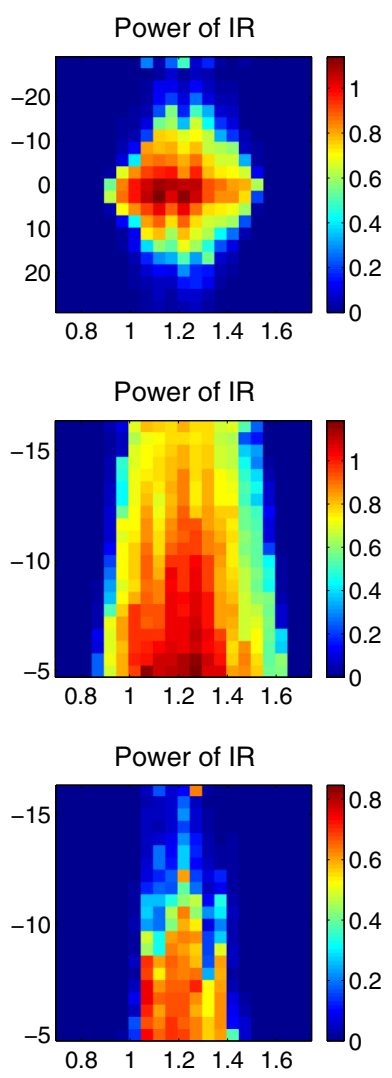

FIG. 12. Two-color lasing phace spaces with the three-undulator configuration and loss curves L3. (a) $N_{B 2}=0.47$ for $368 \mathrm{~nm}$. (b) $N_{B 2}=0.45$ for $368 \mathrm{~nm}$. (c) Fine tuning of $N_{\mathrm{rf}}$ with $N_{B 2}=0.45$ for $368 \mathrm{~nm}$ and more preferable optical axis alignment for IR lasing. (d) Fine tuning of $N_{\text {rf }}$ with $N_{B 2}=0.45$ for $368 \mathrm{~nm}$ and less preferable optical axis alignment for IR lasing. In (a) and (b), the rf detune is scanned from $-0.5 \mathrm{~Hz}$ $\left(N_{\text {rf }}=-27.69\right)$ to $0.5 \mathrm{~Hz}\left(N_{\mathrm{rf}}=27.69\right)$. In (c) and (d), the rf detune is scanned from $-0.29 \mathrm{~Hz}\left(N_{\mathrm{rf}}=-16.06\right)$ to $-0.09 \mathrm{~Hz}$ $\left(N_{\text {rf }}=-4.98\right)$. In all cases, the IR and UV lasing wavelengths are about $695 \mathrm{~nm}$ and $368 \mathrm{~nm}$, respectively, and $N_{B 3}=0$. The beam current is maintained between $19.99 \mathrm{~mA}$ and $21.13 \mathrm{~mA}$.

single-color lasing (outside). The width of this region is determined by the settings of other tuning knobs as well as the beam current. Figure 11 shows the power dependence of two-color lasing on the rf detune and setting of buncher B1 and B3 in the four-undulator configuration, where the rf detune (y-axis) is converted to a relative length scale in terms of the lasing wavelength $\left[N_{\mathrm{rf}}=C \cdot\left(\Delta f_{\mathrm{rf}} / f_{\text {rev }}\right) / \lambda_{\text {FEL }}\right.$, where $\mathrm{C}$ is the circumference of the storage ring, $f_{\text {rev }}$ is the

revolution frequency, and $\lambda_{\mathrm{FEL}}$ is the lasing wavelength.]. Neglecting a small shift in $N_{\text {rf }}$ with the scanning $N_{B 1}\left(N_{B 3}\right)$ over a long period of time, the phase space pattern is nearly symmetric about $N_{B 1 \text {,cen }} \simeq 1.30$ in Fig. 11(a) and $N_{B 3 \text {,cen }} \simeq$ 0.65 in Fig. 11(b). The slow shift in $N_{\text {rf }}$ is attributed to the change of FEL cavity length caused by the temperature change during these 20-minute measurements. Additionally, by properly setting the other knobs, phase space patterns with tuning bunchers B1 and B3 are similar, indicating that B1 and B3 play similar roles in the OK-5 optical klystron. Further, the total phase slippage between two undulators in OK-5 optical klystron can be examined by adding up the contribution from all the magnetic devices and drift spaces between them. Since the settings of the other magnetic devices are the same (see the caption of Fig. 11), by adding $N_{B 1 \text {,cen }}$ and the $N_{B 3}$ value used in Fig. 11(a) $\left[N_{B 3 \text {,cen }}\right.$ and the $N_{B 1}$ value used in Fig. 11(b)], it is found the total phase slippage in Fig. 11(a) is larger than that in Fig. 11(b) by unity, consistent with the periodic power modulation by tuning bunchers shown in the previous section. Also, it indicates the power control of two-color lasing is determined by the total phase slippage in the optical klystrons instead of that produced by any single buncher.

Buncher B2 can also be used to achieve the periodic power modulation. However, since it is a shared buncher by the OK-4 and OK-5 optical klystrons, the tuning of buncher B2 causes wavelength shifts in both lasing colors. Therefore, buncher B2 is mainly used as an auxiliary knob for the precise power control of two-color lasing. Especially, in the three-undulator configuration, by properly setting buncher $B 2$ the OK-4 FEL can lase at the edge of the gain spectrum of the OK-4 optical klystron so that the gain of the OK-4 FEL (UV lasing) is reduced to match that of the OK-5 FEL. Two sets of phase space plots of the two-color lasing with different $N_{B 2}$ values are shown in Fig. 12. Compared to that in Fig. 12(a), $N_{B 2}$ in Fig. 12(b) is set so that the gain of the UV lasing is further reduced, as shown by the enlarged phase space area of IR lasing. Figures 12(c) and 12(d) show the phase spaces of two-color lasing with finer tuning of the rf detune and the same buncher settings as in Fig. 12(b) but different optical axis alignments by tuning the FEL mirrors, which are set more preferable for the IR lasing in Fig. 12(c). It demonstrates that the optical axis alignment is also essential for the control of two-color lasing.

\section{TEMPORAL STRUCTURE}

The study of the dc power stability of two-color lasing shows that the FEL powers at two lasing wavelengths can be maintained stable over an extended period of time with active tuning of the operational parameters. In particular, the lasing powers of two colors are mutually coupled so that the total FEL power of the two colors is more stable than the power of either color during the two-color operation (see Fig. 9). However, the laser beams produced by a storage ring oscillator FEL are not pure dc. Instead, they have two 


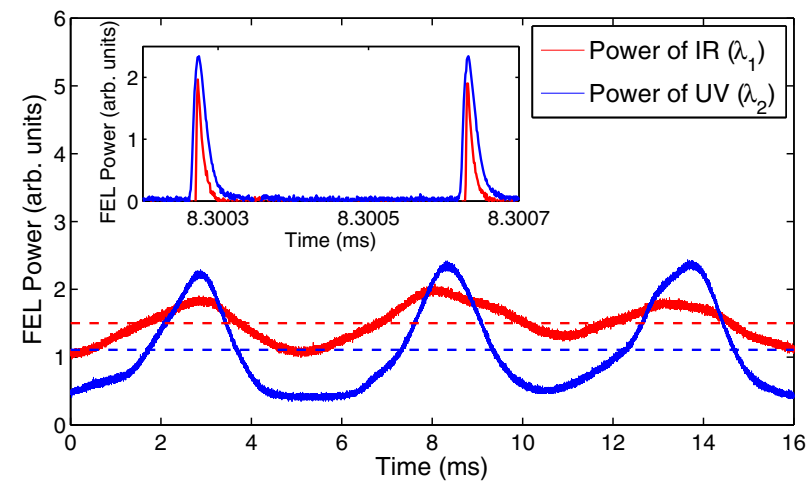

FIG. 13. Short-term (16 milliseconds) power data of the twocolor FEL beams in the three-undulator configuration (Run 2). The sampling rate is $1 \mathrm{GS} / \mathrm{s}$. Two micropulses with the separation of about $358 \mathrm{~ns}$ (equal to the electron revolution period) are shown in the inset. In the main figure, the macropulse structure is shown by plotting the peaks of all the micropulses. The horizontal dashed lines show the mean power of each color. The rf detune is $-0.4 \mathrm{~Hz}$, and the beam current is about $16 \mathrm{~mA}$.

natural temporal structures [58-61]: (1) micropulse structure with the pulse separation equal to the electron revolution period for a single-bunch electron beam; (2) macropulse structure caused by the competition between the electron beam energy damping and FEL induced energy spread increase. The repetition frequency of the macropulses is related to the intrinsic time constants of the storage ring FEL [58],

$$
f_{\text {macro }} \propto \frac{1}{\sqrt{\tau_{\mathrm{FEL}} \tau_{\mathrm{d}}}}
$$

where $\tau_{\mathrm{FEL}}$ is the FEL rise time and $\tau_{\mathrm{d}}$ is the electron beam energy damping time. The macropulses are typically separated by a few to tens of milliseconds for the Duke FEL, much longer than the electron revolution period. These two temporal structures of two-color lasing are studied using two cross-calibrated photodiodes and a fast digital oscilloscope.

As shown in Fig. 13, the temporal structures of two-color FEL beams were measured at a sampling rate of 1 giga samples per second (GS/s) during a well-tuned two-color FEL operation with the FEL micropulses shown in the inset. The temporally overlapped micropulses provide a direct proof that the lasings of two colors occur at the same time. It should be noticed that the duration of the micropulses shown in the figure is determined by the response time of the photodiodes and thus, cannot be directly used to represent the actual duration of FEL micropulses, which is about tens of picoseconds in our case. The peaks of all the micropulses are selected and plotted in the main figure of Fig. 13, showing the FEL macropulse structure. The macropulses of two colors are also synchronized well with roughly simultaneous power rises and decays (with a common repetition time constant of about $5.3 \mathrm{~ms}$ ).
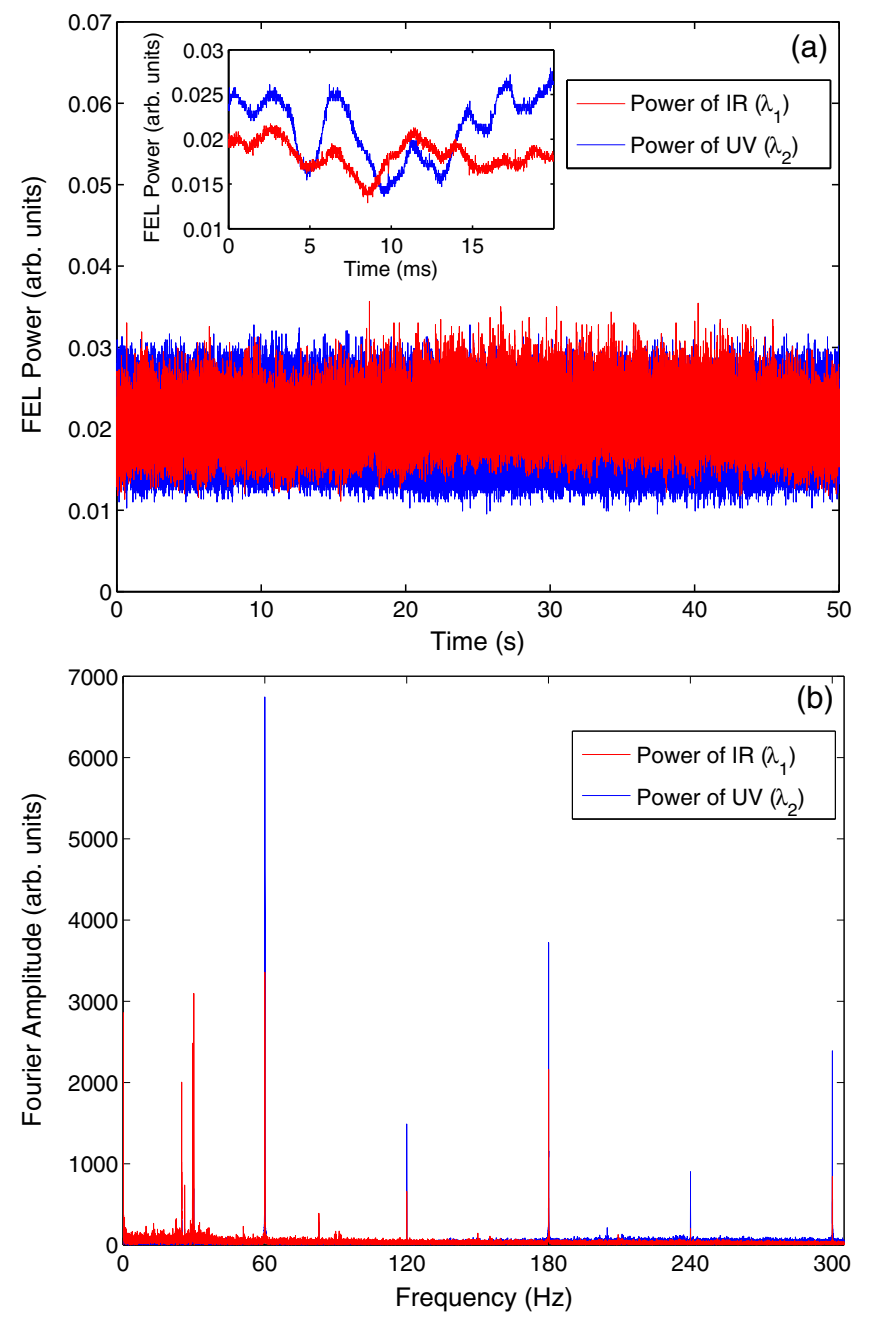

FIG. 14. Longer-term (50 seconds) power data of two-color lasing in the three-undulator configuration (Run 2). (a) Raw power data with a sampling rate of $100 \mathrm{kS} / \mathrm{s}$. The inset is a zoomin plot showing the details of FEL macropulses. (b) Amplitude of the Fourier transform of the same data plotted for the lowfrequency range from 0 to $305 \mathrm{~Hz}$. The rf detune is $-0.4 \mathrm{~Hz}$, and the beam current is about $16 \mathrm{~mA}$.

In addition to the macropulse structure, there is a substantial dc component in the power signals. For the IR lasing, the variation of power level is small with $P_{\max } / P_{\min } \simeq 2.1$; While the UV lasing sees a larger power variation with $P_{\max } / P_{\min } \simeq 6.9$ during the same 16-millisecond measurement period.

Figure 14 shows the power data of a 50-second power measurement with a sampling rate of 100 kilo samples per second $(\mathrm{kS} / \mathrm{s})$. By investigating the power data in the frequency domain [Fig. 14(b)], it is found that the FEL macropulse structures are modulated by the ac line power at $60 \mathrm{~Hz}$ and a few of its harmonics such as 120, 180 and $300 \mathrm{~Hz}$. Using a narrow bandpass filter, the exact frequency and phase of these modulation signals can be determined by analyzing a set of time instants when the modulation is peaked (see the inset of Fig. 15, which shows the $60-\mathrm{Hz}$ 


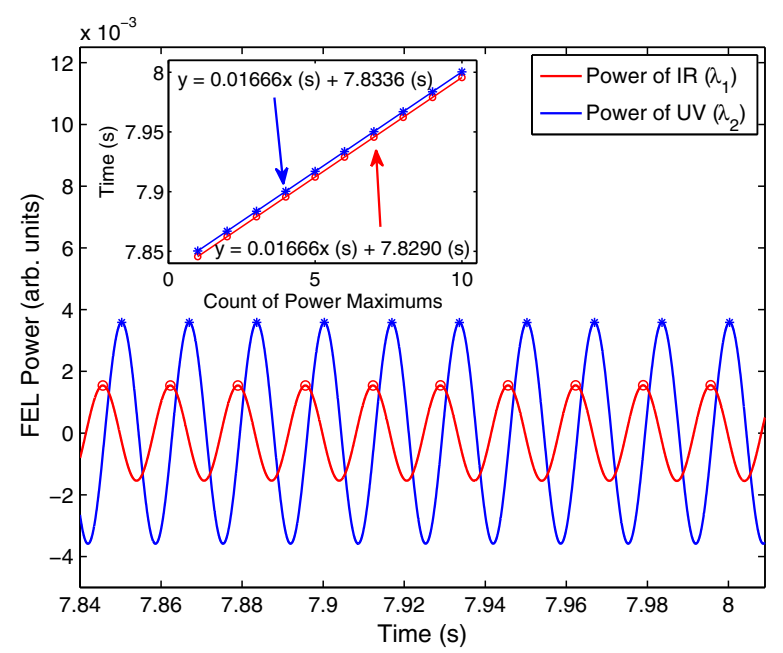

FIG. 15. Filtered 60-Hz ac component of the power data shown in Fig. 14(a) after applying a numerical bandpass filter $(59.54 \mathrm{~Hz}-60.54 \mathrm{~Hz})$. The slopes in the inset represent the period of the ac components $(0.01666 \mathrm{~s}$, corresponding to $60.01 \mathrm{~Hz}$ ), and the intercept difference between two linear fittings (4.7 milliseconds) represents the phase difference $(0.56 \pi \mathrm{rad})$ between this ac component in the two-color power signals.

component as an example). The relative phase between the $60-\mathrm{Hz}$ components of two colors is about $0.56 \pi \mathrm{rad}$ for the selected time window in Fig. 15, and is locked around $0.57 \pi \mathrm{rad}$ with the rms variation of $0.02 \pi \mathrm{rad}$ throughout the entire measurement duration (50 seconds). Regarding the power variations, the power variance $\left(\sigma_{P_{\mathrm{FEL}}}^{2}\right)$ of the 60 $\mathrm{Hz}$ ac component is found to be about $20 \%$ that of the total IR power variance, while for the UV lasing, about $55 \%$ of the power variance is contributed by its $60-\mathrm{Hz}$ ac component. However, the mismatched phase of the $60-\mathrm{Hz}$ ac components between two lasing processes helps decrease the variation of the total lasing power. The other frequency components also contribute to the overall FEL power variations.

\section{SUMMARY}

In this paper, a systematic experimental study of a storage ring two-color FEL is reported. Using the Duke storage ring undulator system with multiple undulators (two OK-4 planar undulators and two OK-5 helical undulators) and a pair of FEL mirrors with two highly reflective wavelength bands, simultaneous two-color lasing with one color in IR and the other in UV has been realized using a common optical cavity. To achieve this two-FEL scheme, two undulator configurations have been utilized and investigated. The first one uses two OK-4 undulators forming an optical klystron FEL to lase at a UV wavelength and one OK-5 undulator, cooperating with one or more downstream radiation sources as a virtual optical klystron, to lase at an IR wavelength. With an additional OK-5 undulator, the second configuration uses a real OK-5 optical klystron sandwiching the OK-4 optical klystron. With the degraded FEL mirrors causing increasing cavity loss, the second undulator configuration with a higher IR gain is more capable of sustaining two-color operation.

For both undulator configurations, wavelength tuning is achieved in multiple ways: (1) tuning the wavelength of one color while keeping the other fixed; (2) tuning both wavelengths simultaneously while keeping them in a second harmonic relation. The wavelength tuning ranges (see Table II) are found to be mainly limited by the highreflectivity bands of the FEL mirrors, which, in our work, are centered around $360 \mathrm{~nm}$ (UV) and $720 \mathrm{~nm}$ (IR). However, we expect that a larger wavelength tuning range can be achieved by using ultra-broadband mirrors with high reflectivity covering the entire visible region. In addition, the power control of two-color lasing has been accomplished using multiple tuning knobs. Among these knobs, the bunchers controlling the phase delay between the optical beams and electron beam (the short time scale), and the rf detune manipulating the synchronization between these beams (the long time scale) are studied using two-dimensional phase space plots. The two-color power stability has been investigated, showing that the FEL powers can be maintained stable for a long period of time with active fine tuning to compensate the change of the accelerator operational conditions. Furthermore, the power change of one color is mostly compensated by that of the other color, and consequently, the total FEL power shows a higher degree of stability.

In addition to dc power measurements, the temporal structures of the FEL beams have been investigated. This study shows that the two-color lasing occurs at the same time with simultaneous IR and UV laser beam micropulses synchronized with the common electron beam circulating in the storage ring. Similar to single-color operation, the two-color lasing displays a quasicontinuous-wave temperal structure with a dc component and a macropulse time structure with pulse separations from a few to tens of milliseconds. The study also shows that the lasing powers of two colors are periodically modulated with a locked phase by the ac line power at $60 \mathrm{~Hz}$ and by its harmonics (120, 180 and $300 \mathrm{~Hz})$.

We plan to continue to study and develop this two-color FEL. One future study will investigate the optical phase relation between two laser beams of different colors. Another area of development is to use this laser to produce a two-color $\gamma$-ray beam at the High Intensity $\gamma$-ray Source (HIGS) $[62,63]$. Using this two-color $\gamma$-ray beam, we will be able to explore new applications in nuclear physics research.

\section{ACKNOWLEDGMENTS}

We would like to thank V. N. Litvinenko for useful discussion and P.W. Wallace for helping improve the 
manuscript. We would also like to acknowledge the engineering and technical staff at DFELL/TUNL for their support. This work is supported by DOE Grant No. DEFG02-97ER41033.

[1] A. Laubereau, in Advances in Laser Spectroscopy, NATO Advances Science Institutes Series, Vol. 95 (Plenum Press, New York and London, 1981), p. 339.

[2] A. Gambetta, G. Galzerano, A. G. Rozhin, A. C. Ferrari, R. Ramponi, P. Laporta, and M. Marangoni, Sub-100 fs twocolor pump-probe spectroscopy of Single Wall Carbon Nanotubes with a $100 \mathrm{MHz}$ Er-fiber laser system, Opt. Express 16, 11727 (2008).

[3] E. Allaria et al., Two-colour pump-probe experiments with a twin-pulse-seed extreme ultraviolet free-electron laser, Nat. Commun. 4, 2476 (2013).

[4] W. M. Tolles, J. W. Nibler, J. R. McDonald, and A. B. Harvey, A review of the theory and application of coherent anti-Stokes Raman spectroscopy (CARS), Appl. Spectrosc. 31, 253 (1977).

[5] F. El-Diasty, Coherent anti-Stokes Raman scattering: Spectroscopy and microscopy, Vib. Spectrosc. 55, 1 (2011).

[6] M. A. Smith, J. W. Hager, and S. C. Wallace, Two-color laser photoionization spectroscopy in a collisionless freejet expansion: spectroscopy and excited-state dynamics of diazabicyclooctane, J. Phys. Chem. 88, 2250 (1984).

[7] L. Matsuoka and S. Hasegawa, Two-color resonance ionization spectroscopy of highly excited titanium atoms, J. Opt. Soc. Am. B 24, 2562 (2007).

[8] C.-S. Friedrich, C. Brenner, S. Hoffmann, A. Schmitz, I. C. Mayorga, A. Klehr, G. Erbert, and M. R. Hofmann, New two-color laser concepts for THz generation, IEEE J. Sel. Top. Quantum Electron. 14, 270 (2008).

[9] M. Tang, H. Minamide, Y. Wang, T. Notake, S. Ohno, and H. Ito, Tunable terahertz-wave generation from DAST crystal pumped by a monolithic dual-wavelength fiber laser, Opt. Express 19, 779 (2011).

[10] E. F. Zalewski and R. A. Keller, Tunable multiple wavelength organic-dye laser, Appl. Opt. 10, 2773 (1971).

[11] H. S. Pilloff, Simultaneous two-wavelength selection in the $\mathrm{N}_{2}$ laser-pumped dye laser, Appl. Phys. Lett. 21, 339 (1972).

[12] C.-Y. Wu and J. R. Lombardi, Simultaneous two-frequency oscillation in a dye laser system, Opt. Commun. 7, 233 (1973).

[13] H. Lotem and R. T. Lynch, Jr., Double-wavelength laser, Appl. Phys. Lett. 27, 344 (1975).

[14] S. G. Bartoshevich, I. V. Mikhnyuk, G. A. Skripko, and I. G. Tarazevich, Efficient difference frequency oscillator based on a Ti:sapphire laser, IEEE J. Quantum Electron. 27, 2234 (1991).

[15] R. Scheps and J. F. Myers, Doubly resonant Ti:sapphire laser, IEEE Photonics Technol. Lett. 4, 1 (1992).

[16] M. R. X. de Barros and P. C. Becker, Two-color synchronously mode-locked femtosecond Ti:sapphire laser, Opt. Lett. 18, 631 (1993).
[17] F. Siebe, K. Siebert, R. Leonhardt, and H. G. Roskos, A fully tunable dual-color $\mathrm{CW} \mathrm{Ti}: \mathrm{Al}_{2} \mathrm{O}_{3}$ laser, IEEE J. Quantum Electron. 35, 1731 (1999).

[18] T. Hidaka and Y. Hatano, Simultaneous two wave oscillation LD using biperiodic binary grating, Electron. Lett. 27, 1075 (1991).

[19] C.-L. Wang and C.-L. Pan, Tunable dual-wavelength operation of a diode array with an external grating-loaded cavity, Appl. Phys. Lett. 64, 3089 (1994).

[20] S. P. Reilly, S. W. James, and R. P. Tatam, Tunable and switchable dual wavelength lasers using optical fibre Bragg grating external cavities, Electron. Lett. 38, 1033 (2002).

[21] X. Liu, D. Han, Z. Sun, C. Zeng, H. Lu, D. Mao, Y. Cui, and F. Wang, Versatile multi-wavelength ultrafast fiber laser mode-locked by carbon nanotubes, Sci. Rep. 3, 2718 (2013).

[22] H. Ahmad, F. D. Muhammad, C. H. Pua, and K. Thambiratnam, Dual-wavelength fiber lasers for the optical generation of microwave and terahertz radiation, IEEE J. Sel. Top. Quantum Electron. 20, 0902308 (2014).

[23] J. M. J. Madey, Stimulated emission of bremsstrahlung in a periodic magnetic field, J. Appl. Phys. 42, 1906 (1971).

[24] L. R. Elias, W. M. Fairbank, J. M. J. Madey, H. A. Schwettman, and T. I. Smith, Observation of Stimulated Emission of Radiation by Relativistic Electrons in a Spatially Periodic Transverse Magnetic Field, Phys. Rev. Lett. 36, 717 (1976).

[25] R. Bonifacio, C. Pellegrini, and L. M. Narducci, Collective instabilities and high-gain regime in a free electron laser, Opt. Commun. 50, 373 (1984).

[26] P. Emma et al., First lasing and operation of an ångstromwavelength free-electron laser, Nat. Photonics 4, 641 (2010).

[27] L.-H. Yu et al., High-Gain Harmonic-Generation FreeElectron Laser, Science 289, 932 (2000).

[28] L. H. Yu et al., First Ultraviolet High-Gain HarmonicGeneration Free-Electron Laser, Phys. Rev. Lett. 91, 074801 (2003).

[29] N. A. Vinokurov and A. N. Skrinsky, Budker Institute of Nuclear Physics, Novosibirsk, Report No. INP 77-59, 1977.

[30] I. B. Drobyazko, G. N. Kulipanov, V. N. Litvinenko, I. V. Pinayev, V. M. Popik, I. G. Silvestrov, A. N. Skrinsky, A. S. Sokolov, and N. A. Vinokurov, Lasing in visible and ultraviolet regions in an optical klystron installed on the VEPP-3 storage ring, Nucl. Instrum. Methods Phys. Res., Sect. A 282, 424 (1989).

[31] D. Nutarelli, D. Garzella, E. Renault, L. Nahon, and M. E. Couprie, Super ACO FEL oscillation at $300 \mathrm{~nm}$, Nucl. Instrum. Methods Phys. Res., Sect. A 445, 143 (2000).

[32] A. A. Lutman, R. Coffee, Y. Ding, Z. Huang, J. Krzywinski, T. Maxwell, M. Messerschmidt, and H.-D. Nuhn, Experimental Demonstration of Femtosecond Two-Color X-Ray Free-Electron Lasers, Phys. Rev. Lett. 110, 134801 (2013).

[33] A. Marinelli, A. A. Lutman, J. Wu, Y. Ding, J. Krzywinski, H.-D. Nuhn, Y. Feng, R. N. Coffee, and C. Pellegrini, Multicolor Operation and Spectral Control in a GainModulated X-Ray Free-Electron Laser, Phys. Rev. Lett. 111, 134801 (2013). 
[34] T. Hara et al., Two-colour hard X-ray free-electron laser with wide tunability, Nat. Commun. 4, 2919 (2013).

[35] D. A. Jaroszynski, R. Prazeres, F. Glotin, and J. M. Ortega, Two-Color Free-Electron Laser Operation, Phys. Rev. Lett. 72, 2387 (1994).

[36] D. A. Jaroszynski, R. Prazeres, F. Glotin, and J. M. Ortega, Two-colour operation of the free-electron laser using a step-tapered undulator, Nucl. Instrum. Methods Phys. Res., Sect. A 358, 224 (1995).

[37] R. Prazeres, F. Glotin, C. Insa, D. A. Jaroszynski, and J. M. Ortega, Two-colour operation and applications of the CLIO FEL in the mid-infrared range, Nucl. Instrum. Methods Phys. Res., Sect. A 407, 464 (1998).

[38] Y. K. Wu, N. A. Vinokurov, S. Mikhailov, J. Li, and V. Popov, High-Gain Lasing and Polarization Switch with a Distributed Optical-Klystron Free-Electron Laser, Phys. Rev. Lett. 96, 224801 (2006).

[39] M. Mende, S. Günster, H. Ehlers, and D. Ristau, Optical Properties of Ion Beam Sputtered Oxide Mixture Coatings, in Optical Interference Coatings (Optical Society of America, Tucson, Arizona, 2010), p. ThA4.

[40] M. F. Velghe, M. E. Couprie, and M. Billardon, Specific optical properties of multilayer mirrors for FEL experiments, Nucl. Instrum. Methods Phys. Res., Sect. A 296, 666 (1990).

[41] A. Gatto et al., Multiscale degradations of storage ring FEL optics, Nucl. Instrum. Methods Phys. Res., Sect. A 483, 172 (2002).

[42] P. D. Townsend, Colour centres past, present and future, Nature (London) 258, 293 (1975).

[43] K. Boller, R.-P. Haelbich, H. Hogrefe, W. Jark, and C. Kunz, Investigation of carbon contamination of mirror surfaces exposed to synchrotron radiation, Nucl. Instrum. Methods Phys. Res. 208, 273 (1983).

[44] D. Garzella, M. E. Couprie, T. Hara, L. Nahon, M. Brazuna, A. Delboulbé, and M. Billardon, Mirror degradation and heating in storage ring FELs, Nucl. Instrum. Methods Phys. Res., Sect. A 358, 387 (1995).

[45] S. F. Mikhailov, V. G. Popov, J. Li, and Y. K. Wu, Pass-byPass Multistage FEL Gain Measurement Technique for a Storage Ring FEL, in Proceedings of the 23rd Particle Accelerator Conference, Vancouver, Canada, 2009 (IEEE, Piscataway, NJ, 2009), p. 1241.

[46] M. Billardon, M. E. Couprie, J. M. Ortega, and M. Velghe, Fabry-Perot effects in the exponential decay and phase shift reflectivity measurement methods, Appl. Opt. 30, 344 (1991).

[47] Y. K. Wu, J. Yan, H. Hao, J. Y. Li, S. F. Mikhailov, V. G. Popov, N. A. Vinokurov, S. Huang, and J. Wu, Widely Tunable Two-Color Free-Electron Laser on a Storage Ring, Phys. Rev. Lett. 115, 184801 (2015).

[48] P. Elleaume, Microtemporal and spectral structure of storage ring free-electron lasers, IEEE J. Quantum Electron. 21, 1012 (1985).
[49] V. N. Litvinenko and N. A. Vinokurov, Lasing spectrum and temporal structure in storage ring FELs: Theory and experiment, Nucl. Instrum. Methods Phys. Res., Sect. A 304, 66 (1991).

[50] G. S. Brown, V. P. Suller, and E. Zinin, Measurement of bunch length with an image dissector tube, IEEE Trans. Nucl. Sci. 30, 2348 (1983).

[51] N. A. Vinokurov and A. N. Skrinsky, Budker Institute of Nuclear Physics, Novosibirsk, Report No. INP 77-67, 1977.

[52] A. Renieri, Storage ring operation of the free-electron laser: The amplifier, Il Nuovo Cimento Soc. Ital. Fis. 53B, 160 (1979).

[53] P. Elleaume, Storage ring FEL theory, Nucl. Instrum. Methods Phys. Res., Sect. A 237, 28 (1985).

[54] V. N. Litvinenko, S. H. Park, I. V. Pinayev, and Y. $\mathrm{Wu}$, Performance of the OK-4/Duke storage ring FEL, Nucl. Instrum. Methods Phys. Res., Sect. A 470, 66 (2001).

[55] B. Jia, J. Li, S. Huang, S. C. Schmidler, and Y. K. Wu, Electron beam energy spread measurements using optical klystron radiation, Phys. Rev. Accel. Beams 13, 080702 (2010).

[56] B. Jia, Study of Storage Ring Free-Electron Laser Using Experimental and Simulation Approaches, Ph.D. thesis, Duke University, 2011.

[57] S. Huang, J. Li, and Y. K. Wu, A Direct Electron Beam Energy Spread Measurement System for Beam Instability and FEL Research, in Proceedings of the 22nd Particle Accelerator Conference, PAC-2007, Albuquerque, NM (IEEE, New York, 2007), p. 4045.

[58] M. Billardon, Storage Ring Free-Electron Laser and Chaos, Phys. Rev. Lett. 65, 713 (1990).

[59] M. Billardon, D. Garzella, and M. E. Couprie, Saturation Mechanism for A Storage-Ring Free-Electron Laser, Phys. Rev. Lett. 69, 2368 (1992).

[60] M. E. Couprie and P. Elleaume, Modification of the electron bunch shape induced by the Orsay storage ring FEL, Nucl. Instrum. Methods Phys. Res., Sect. A 259, 77 (1987).

[61] H. Hama, K. Kimura, J. Yamazaki, S. Takano, T. Kinoshita, and M.-E. Couprie, Microscopic study on lasing characteristics of the UVSOR storage ring free electron laser, Nucl. Instrum. Methods Phys. Res., Sect. A 375, 32 (1996).

[62] V. N. Litvinenko et al., Gamma-Ray Production in a Storage Ring Free-Electron Laser, Phys. Rev. Lett. 78, 4569 (1997).

[63] H. R. Weller, M. W. Ahmed, H. Gao, W. Tornow, Y. K. Wu, M. Gai, and R. Miskimen, Research opportunities at the upgraded HI $\gamma$ S facility, Prog. Part. Nucl. Phys. 62, 257 (2009). 